\title{
Vibration analysis of circular cylindrical double-shell structures under general coupling and
}

\author{
end boundary conditions \\ Chunyu Zhang*, Guoyong Jin, Xianglong Ma and Tiangui Ye \\ College of Power and Energy Engineering, Harbin Engineering University, Harbin, 150001, P. R. China
}

\begin{abstract}
In this paper, the free and forced vibration analysis of circular cylindrical double-shell structures under arbitrary boundary conditions is presented. This is achieved by employing the improved Fourier series method based on Hamilton's principle. In the formulation, each displacement component of the cylindrical shells and annular plates is invariantly expanded as the superposition of a standard Fourier series with several supplementary functions introduced to remove the potential discontinuities of the original displacement and its derives at the boundaries. With the introduction of four sets of boundary springs at the coupling interfaces and end boundaries of the shell-plate combination, both elastic and rigid coupling and end boundary conditions can be easily obtained by assigning the stiffnesses of the artificial springs to certain values. The natural frequencies and mode shapes of the structures as well as frequency responses under forced vibration are obtained with the Rayleigh-Ritz procedure. The convergence of the method is validated by comparing the present results with those obtained by the finite element method. Several numerical results including natural frequencies and mode shapes are presented to demonstrate the excellent accuracy and reliability of the current method. Finally, a number of parameter studies concerning various end and coupling boundary conditions, different dimensions of shells and annular plates are also performed.
\end{abstract}

Keywords: vibration; double-shell structure; general boundary conditions; improved Fourier series

* Corresponding author, Tel:+86 451 8289199; fax: +96 451 82518264. E-mail address:hdzhangchunyu@163.com, guoyongjin@hrbeu.edu.cn 


\section{Introduction}

The cylindrical shell structures, as a type of fundamental structural components, are widely used in the engineering applications such as missiles, submarine hulls, oil-transmitting pipelines. In practice, this kind of structures is often subjected to the intricate environment and dynamic loads which can result in violent vibration, fatigue damage and radiating noise. Thus, it is of great importance to achieve an insight into the dynamic and vibration characteristics of the cylindrical shell structures for the engineers.

In the past several decades, a huge number of analytical and numerical methods have been conducted to analyze the vibration behaviors of various shell structures [1-8]. Most of the studies were confined only to the problems of single cylindrical shells, however, in practical engineering there are many limitations in the application of the single cylindrical shell for its simplex structural property. Therefore, several kinds of redesigned structures like ring-stiffened cylindrical shell, shellplate combination systems and conical-cylindrical shells combination systems cause more and more scholars' attention. Reinforcing shells with stiffener rings is an efficient way to enhance the stiffness of a structure without adding much mass, as a result, increasingly researchers focused on rings-stiffened cylindrical shells. These stiffening ring elements slightly increase the structure weight, but have apparent effects on the structural stability and strength. Depending on different methods taking account of the effects of circumferential ring stiffeners, two kinds of approaches are usually employed. Firstly, the ring stiffeners are evaluated the by averaging their properties over the surface of the shell and its thickness is suitably modified, which is usually called as smeared approach. For example, Hopmann [9] presented the free vibration analysis of simply supported orthogonally stiffened cylindrical shells analytically based on smearing method. Mikulas and McElman [10] investigated the effects of stiffener eccentricities on the buckling characteristics of eccentrically 
stiffened simply supported cylindrical shells by averaged the stiffeners properties over the surface of the shells. Chen et al. [11] studies the vibrational behavior and far-field sound radiation of a submerged stiffened conical shell in which the smearing method was used to model the ring stiffeners. However the smeared approach is limited to shells with the stiffened ring equally spaced. For a more general model, the ring stiffeners have to be treated as discrete elements and many methods have been proposed by researchers. Jafari and Bagheri [12] presented an energy method, and Mustafa and Ali [13] employed the Rayleigh-Ritz procedure to investigate the free vibration of cylindrical shells stiffened by non-uniform circumferential stiffeners. Wei et al. [14] presented a wave based method to analysis the free vibration of cylindrical shells with non-uniform stiffener distributions, in which the stiffener are modeled as annular plates and coupled with the cylindrical shells according to the force and displacement continuity. Except circumferential ring stiffeners, many researchers [15-16] also focused on the effects of longitudinal ribs stiffeners on the vibration characteristics of cylindrical shells. Wang and Rinehart [17] concerned with the free-vibration characteristic of thin cylindrical shells reinforced by longitudinal stiffeners, and the structural system was treated as an isotropic cylinder interacting of a set of discrete thin walled stiffeners. Averbukh and Mavlyutov [18] studied the vibration behaviors of a cylindrical shell with longitudinal ribs in which the relationships between the displacements and forces on the contact lines were established by means of the Green's matrix of vibrations of the shell and Green's function of the vibrations of the rod. Vanlaere et al. [19] considered both the effects of longitudinal stiffeners and circumferential rings on the strength and vibration characteristics of the cylindrical shell. In addition to the vibration problem, numerous works have been devoted to the analysis the sound radiation of cylindrical shells and most of the researches focused on shells under water or fluid-filled [20-22]. 
Another kind of redesigned structures like cylinder-plate combinations, cylindrical-conical shells, and cylindrical-spherical shells also got the attentions of several researchers, but not too many. Tso and Hansen [23] investigated the wave propagation characteristics through cylindrical-plate junctions, in which equilibrium and compatibility condition were applied to the junction to evaluate the wave amplitude and hence the wave powers. Cheng and Nicolas [24] presented a theoretical study on a plate-ended circular cylindrical shell radiating sound into its enclosed cavity. Later, based on the same method Cheng [25] extended his work to study the internal sound field of the plate-ended circular cylindrical shell. Chen et al. [26] studied the vibration behavior of ring stiffened cylindrical shell with intermediate large frame ribs based on the wave based method. The model is subdivided into cylindrical shells, ring stiffeners and large ribs whose vibration fields are expanded by wave functions which exactly satisfy the governing equations of the motions. Qu et al. [27] developed a modified variational method for dynamic analysis of ring-stiffened conical-cylindrical shells subjected to different boundary conditions. Wu et al. [28] proposed a domain decomposition technique for solving free and forced vibrations of spherical-cylindrical-spherical shell, based on Reissner-Naghdi's thin shell theory. Yan and Zhang [29] studied the vibrational power flow in a submerged infinite cylindrical shell with internal rings and bulkheads was analytically. The coupling condition on the outer surface of the shell wall was introduced to obtain the vibrational equations of this coupled system.

From the literature review, it appears that the double-shell structures are seldom attempted. However, the double-shell structures is a kind of widely used and important engineering structures due to their superior mechanical properties and low vibration and noise features. To the authors' best knowledge, the only paper available in the literature is that of Yamada et al. [30] concerning on the free vibration of a circular cylindrical double-shell system closed by end plates. The main reason may 
lie in the degree complexity of the governing shell equations and the difficulties associated with matching the interface coupling conditions between the substructures. In addition, it is worth pointed that the end boundary conditions of the shells in the above mentioned literature are mostly preassigned, and many methods are limited to certain classical end boundary conditions like simply supported or clamped supported. Nevertheless, in engineering practice, the end boundary conditions of the double-shell combinations may not be fixed in a certain classical restraint, a variety of end boundary conditions like elastic boundaries, point-supported ones, or other combined boundary conditions may be encountered. So far, the study of the vibration of shell structures with complex geometries and general boundary conditions is mainly carried out by traditional approximate methods, in particular by the finite element method (FEM). The FEM is certainly one of the most powerful computational methods to manage complex geometries and boundary conditions. But, there are two main shortcomings of the FEM, firstly, the accuracy of FEM is dependent on the mesh size of the discrete element and high accuracy can easily result in unacceptable computational cost and saturation of memory resources; secondly, it is cumbersome to treat the elastic coupling and end boundary conditions in FEM and the FE mesh should be re-meshed frequently when the structural dimensions are changed in paramedical study. Consequently, it is of great significance to develop an effective and accurate solution approach for the vibration problems of the double-shell structures with general boundary conditions.

The main purpose of this work is to develop an alternative and accurate analytical method for the vibration analysis of circular cylindrical double-shell structure coupled and reinforced with annular plates with general boundary conditions and to present some useful results for the future researchers. The improved Fourier series method is employed, which was originally proposed by Li [31] for the vibration analysis of beams. Subsequently, this method is exploited to research the 
vibration behaviors of plates [32, 33], cylindrical shells [7]. Under the framework, regardless of boundary conditions, the displacements of the cylindrical shells and annular plates are invariantly represented as a Fourier series with closed-form supplementary functions. The introduction of the supplementary functions can not only remove the potential discontinuities at the joints and end boundaries but also ensure and accelerate the convergence of the expansion series. The artificial spring system is employed to formulate the boundary and coupling conditions. The general elastic boundary and coupling conditions can be obtained easily by changing the values of the artificial springs. Since the displacement field is constructed adequately smooth throughout the entire solution domain, an exact solution can be obtained by employing Rayleigh-Ritz procedure based upon the energy functions of the shell-plate combination. The organization of this paper is as follows. The first part of this paper reviews the work already done about vibration analysis if cylindrical shell structures. In second part, the model description and theoretical formulation of the circular cylindrical doubleshell structures are implemented. Then free and forced vibration results of double-shell structure are presented and discussed in part three. Convergence study, validation and parameter study for free vibration analysis are first conducted. Then the forced vibration responses are given by comparing the results from the finite element program ANSYS. The effects of the dimensions of the annular plates on the dynamic responses are also presented. Finally, concluding remarks are made in forth part.

\section{Theoretical formulation}

\subsection{Model description}

The geometry of a typical circular cylindrical double-shell structure and the co-ordinate system related to each shell and plate component are depicted in Fig.1. The double-shell structure is composed of two concentric circular cylindrical shells which are coupled and reinforced by $N$ number 
of unevenly distributed annular plates along the axial direction. A cylindrical coordinate system $(o, r$, $\theta, x)$ is considered for each shell component, in which $R_{i}, h_{i}$ and $L_{i}$ denote the radius, thickness and length of the inner cylindrical shell, respectively, $R_{o}, h_{o}$ and $L_{o}$ represent those of the outer one. The reinforced annular plates are assumed to be of uniform thickness $h_{p}$ and described with the $o-r, s, \theta$ coordinate system, in which $s$ is measured along the radial direction from its inner edge to the outer edge. $b\left(b=R_{\mathrm{o}}-R_{i}\right)$ is the width of the annular plate. The $n$th annular plate is arranged at $x_{n}$ which is measured from the left end of the shells. The displacement components of the cylindrical shell and annular plate with respect to their corresponding coordinate systems are described as $u_{\delta}, v_{\delta}$ and $w_{\delta}(\delta=s, p)$, in which the subscripts $s$ and $p$ represent the cylindrical shell and annular plate respectively. The thicknesses of the components are assumed to be uniform and negligible compared to lengths or radii. As a result, the thin plate and shell theories are applicable in the following procedure.

\subsection{Energy functions of cylindrical shell and annular plate}

In this paper, the weak form of solutions are sought by using the Rayleigh-Ritz energy method. Consequently, the energy functional of the structure are derived at first. The entire energy functional $\Pi_{l}$ for the structure includes the stain energy and kinetic energy for the annular plates and cylindrical shells, expressed as

$$
\Pi_{l}=\sum_{r=1}^{N_{r}}\left(T_{p}^{r}-U_{p}^{r}\right)+\sum_{k=1}^{2}\left(T_{s}^{k}-U_{s}^{k}\right)
$$

where the $T_{p}^{r}$ and $U_{p}^{r}$ are the kinetic and strain energy of the $r$ th annular plate and $T_{s}^{k}$ and $U_{s}^{k}$ represent those of the $k$ th cylindrical shell, respectively. $N_{r}$ indicates the number of the annular plates. The formulations of $T_{p}^{r}, U_{p}^{r}, T_{s}^{k}$ and $U_{s}^{k}$ will be derived separately in the following procedures. 
With regard to the annular plate components, based on the thin plate theory [1], the straindisplacement relations can be described as following with the consideration of both in-plane and outplane vibrations

$$
\begin{aligned}
\varepsilon_{s}^{p} & =\frac{\partial u_{p}^{r}}{\partial s} \\
\varepsilon_{\theta}^{p} & =\frac{1}{s+a}\left(\frac{\partial v_{p}^{r}}{\partial \theta}+u_{p}^{r}\right) \\
\gamma_{s \theta}^{p} & =\frac{\partial v_{p}^{r}}{\partial s}+\frac{1}{s+a}\left(\frac{\partial u_{p}^{r}}{\partial \theta}-v_{p}^{r}\right) \\
\chi_{s}^{p} & =-\frac{\partial^{2} w_{p}^{r}}{\partial s^{2}} \\
\chi_{\theta}^{p} & =-\frac{1}{(s+a)^{2}} \frac{\partial^{2} w_{p}^{r}}{\partial \theta^{2}}-\frac{1}{s+a} \frac{\partial w_{p}^{r}}{\partial s} \\
\tau_{s \theta}^{p} & =-\frac{2}{s+a} \frac{\partial^{2} w_{p}^{r}}{\partial s \partial \theta}+\frac{2}{(s+a)^{2}} \frac{\partial w_{p}^{r}}{\partial \theta}
\end{aligned}
$$

where $\varepsilon_{s}^{p}, \varepsilon_{\theta}^{p}$ and $\gamma_{s \theta}^{p}$ are the normal and shear strains in the middle surface. $\chi_{s}^{p}$ and $\chi_{\theta}^{p}$ donate the mid-surface changes in curvature and $\tau_{s \theta}^{p}$ represents the mid-surface twist. Therefore, the full expressions of the strain energy $V_{p}^{r}$ and the kinetic energy $T_{p}^{r}$ for the $r$ th annular plate can be written as

$$
\begin{aligned}
V_{p}^{r} & =\frac{E_{p} h_{p}}{2\left(1-v_{p}^{2}\right)} \int_{0}^{2 \pi} \int_{0}^{b}\left\{\left(\varepsilon_{s}^{p}\right)^{2}+\left(\varepsilon_{\theta}^{p}\right)^{2}+2 \mu_{p} \varepsilon_{s}^{p} \varepsilon_{\theta}^{p}+\frac{1-v_{p}}{2}\left(\gamma_{s \theta}^{p}\right)^{2}\right\}(s+a) \mathrm{d} s \mathrm{~d} \theta \\
& +\frac{E_{p} h_{p}^{3}}{24\left(1-v_{p}^{2}\right)} \int_{0}^{2 \pi} \int_{0}^{b}\left\{\left(\chi_{s}^{p}\right)^{2}+\left(\chi_{\theta}^{p}\right)^{2}+2 \mu_{p} \chi_{s}^{p} \chi_{\theta}^{p}+\frac{1-v_{p}}{2}\left(\tau_{s \theta}^{p}\right)^{2}\right\}(s+a) \mathrm{d} s \mathrm{~d} \theta \\
T_{p}^{r} & =\frac{\rho_{p} h_{p}}{2} \int_{0}^{2 \pi} \int_{0}^{b}\left\{\left(\frac{\partial u_{p}^{r}}{\partial t}\right)^{2}+\left(\frac{\partial v_{p}^{r}}{\partial t}\right)^{2}+\left(\frac{\partial w_{p}^{r}}{\partial t}\right)^{2}\right\}(s+a) \mathrm{d} s \mathrm{~d} \theta
\end{aligned}
$$


where $E_{p}, r_{p}$ and $m_{p}$ denote the Young's modulus, the mass density and the Poisson ratio of the plate, respectively.

For the $k$ th cylindrical shell component, the strains, curvatures and twist changes based on the Goldenveizer-Novozhilov shell theory [1] can be expressed as

$$
\begin{aligned}
& e_{x}^{s}=\frac{\llbracket u_{s}^{k}}{\llbracket x}
\end{aligned}
$$

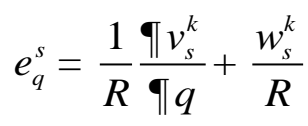

$$
\begin{aligned}
& e_{x q}^{s}=\frac{1}{R} \frac{\llbracket u_{s}^{k}}{\| q}+\frac{\llbracket v_{s}^{k}}{\llbracket x} \\
& c_{x}^{s}=-\frac{\mathbb{I}^{2} w_{s}^{k}}{\llbracket x^{2}}
\end{aligned}
$$

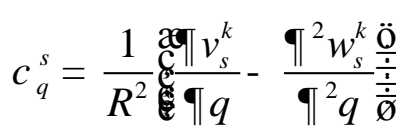

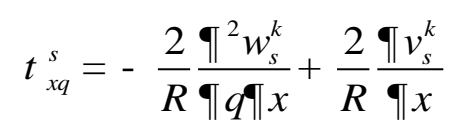

Consequently, the strain and the kinetic energy expressions $V_{s}^{k}, T_{s}^{k}$ for the $k$ th cylindrical shell can be written as

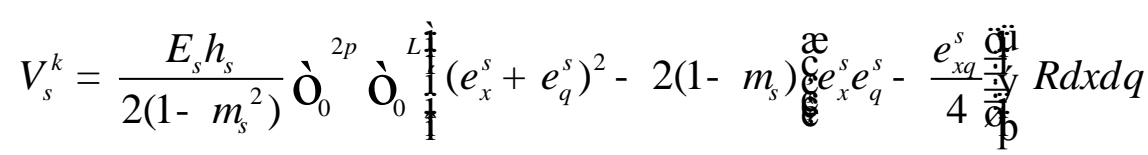

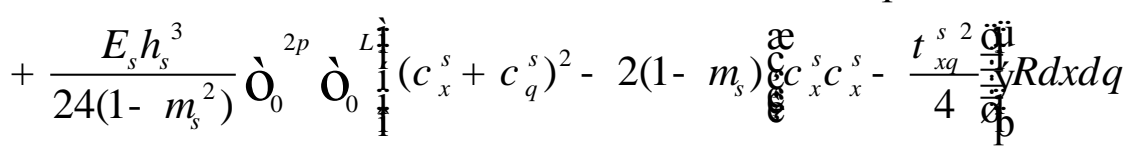

$$
\begin{aligned}
& T_{s}^{k}=\frac{\rho_{s} h_{s}}{2} \int_{0}^{2 \pi} \int_{0}^{L}\left\{\left(\frac{\partial u_{s}^{k}}{\partial t}\right)^{2}+\left(\frac{\partial v_{s}^{k}}{\partial t}\right)^{2}+\left(\frac{\partial w_{s}^{k}}{\partial t}\right)^{2}\right\} R \mathrm{~d} x \mathrm{~d} \theta
\end{aligned}
$$

where $E_{s}, \mu_{s}$ and $\rho_{s}$ denote the Young's modulus, Poisson ratio and density of the cylindrical shell. 
In practice, the structures are often subjected to an intricate environment, and the excitations come from outside are inevitable. Therefore, the forced vibration analysis of the structures deserves particular attention. For simplicity, only a point force applied on the inner shell in the radial direction is taken into consideration in this paper, then the energy function $\tilde{O}_{F}$ for the point force can be expressed as

$$
\Pi_{F}=\int_{0}^{L} \int_{0}^{2 \pi}\left[F(x, \theta) \cdot w_{s}^{2}(x, \theta)\right] R_{i} \mathrm{~d} \theta \mathrm{d} x
$$

The point force applied on the shell surface can be expressed as

$$
F(x, \theta)=F_{0} \delta\left(x-x_{F}\right) \delta\left(\theta-\theta_{F}\right)
$$

where $\delta()$ is the Dirac function, $\left(x_{F}, \theta_{F}\right)$ indicate the coordinates of the point force, and $F_{0}$ is the amplitude of the point force.

\subsection{Boundary conditions}

In order to meet the requirements of different boundary conditions in engineering practices, a general method which can be used in dealing with arbitrary boundary conditions is of practical significance. The present study is devoted to establish a unified numerical method which could satisfy the above mentioned requests. It should be stressed that there are two categories of boundary conditions involved. The first one is the end boundary conditions at the ends of the cylindrical shells and the second part is the coupling boundary conditions at the junctions of the cylindrical shells and annular plates. In this paper, the arbitrary boundary conditions of the double-shell structures are implemented by the aid of artificial boundary spring technique. Thus, the desired coupling and end boundary conditions can be obtained by assigning the stiffnesses of the artificial springs to certain values. Specially, at each boundary of the structures, three sets of linear springs and one set of 
rotational spring which are continuously distributed along the boundaries are introduced separately to simulate the boundary and continuity conditions.

In Fig.2, $k_{x k}^{\gamma}, k_{r k}^{\gamma}$ and $k_{\theta k}^{\gamma}$ represent the stiffnesses of the linear spring sets used to restrain the displacement in axial, radial and circumferential directions, respectively, and $K_{r k}^{\gamma}$ is the stiffness for the rotational springs used to restrain the rotations, where the superscript $\gamma=R, L, c_{i}$ indicates the axial position of the springs at the right end, left end and coupling junctions of the structures. The subscript $k$ equal 1 and 2 separately represent the boundaries on the outer and inner cylindrical shells.

Consequently, the boundary conditions at the ends of the cylindrical shells can be expressed in terms of boundary spring components as

$$
\begin{array}{ll}
k_{x k}^{L} u_{s}^{k}-\left.N_{x, s}^{k}\right|_{x=0}=0, & k_{\theta k}^{L} v_{s}^{k}-\left.N_{x \theta . s}^{k}\right|_{x=0}=0 \\
k_{r k}^{L} w_{s}^{k}-Q_{x, s}^{k}-\left.\frac{\partial M_{x \theta, s}^{k}}{R \partial \theta}\right|_{x=0}=0, & K_{r k}^{L} \frac{\partial w_{s}^{k}}{\partial x}+\left.M_{x, s}^{k}\right|_{x=0}=0 \\
k_{x k}^{R} u_{s}^{k}-\left.N_{x, s}^{k}\right|_{x=L}=0, & k_{\theta k}^{R} v_{s}^{k}-\left.N_{x \theta . s}^{k}\right|_{x=L}=0 \\
k_{r k}^{R} w_{s}^{k}-Q_{x, s}^{k}-\left.\frac{\partial M_{x \theta, s}^{k}}{R \partial \theta}\right|_{x=L}=0, & K_{r k}^{R} \frac{\partial w_{s}^{k}}{\partial x}+\left.M_{x, s}^{k}\right|_{x=L}=0
\end{array}
$$

where $N_{x, s}^{k}$ and $N_{x \theta, s}^{k}$ indicate the two in-plane forces, $Q_{x, s}^{k}$ is the transverse shear force, and $M_{x, s}^{k}$ and $N_{x \theta, s}^{k}(k=1,2)$ represent the bending and twisting moments, respectively. $N_{x, s}^{k}, N_{x \theta, s}^{k}, Q_{x, s}^{k}, M_{x, s}^{k}$, and $M_{x \theta, s}^{k}$ are the force and moment resultants of the $k$ th cylindrical shell component which can be obtained by integrating the stresses over the shell thickness, expressed as

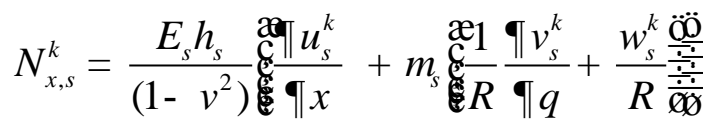

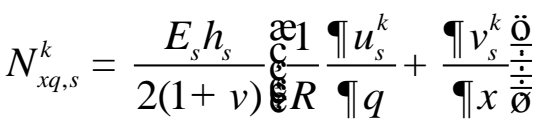




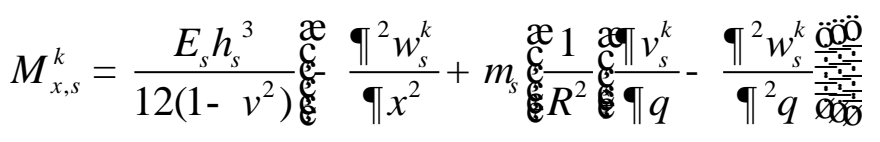

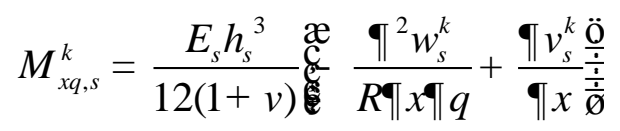

$$
\begin{aligned}
& Q_{x, s}^{k}=\frac{\| M_{x, \mathrm{~s}}^{k}}{\mathbb{\|} x}+\frac{\boldsymbol{\Phi} M_{x q, s}^{k}}{R \| q}
\end{aligned}
$$

At the coupling interface of the $r$ th annular plate and cylindrical shells, the continuity and equilibrium boundary conditions can be expressed as

$$
\begin{aligned}
& k_{x k}^{c_{r}}\left(u_{s}^{k}-w_{p}^{r}\right)-\left.N_{x, s}^{k}\right|_{x=x_{c}}=0 \\
& k_{\theta k}^{c_{r}}\left(\mathrm{v}_{s}^{k}-v_{p}^{r}\right)-\left.N_{x \theta, \mathrm{s}}^{k}\right|_{x=x_{c}}=0 \\
& k_{r k}^{c_{r}}\left(\mathrm{w}_{s}^{k}-u_{p}^{r}\right)-\left.Q_{x, s}^{k}\right|_{x=x_{c}}=0 \\
& K_{r k}^{c_{r}}\left(\frac{\partial w_{s}^{k}}{\partial x}-\frac{\partial w_{p}^{r}}{\partial s}\right)-\left.M_{x, s}^{k}\right|_{x=x_{c}}=0
\end{aligned}
$$

where $x_{c}$ represents the axial position of the annular plate.

Finally, the corresponding potential stored in the end boundaries and shell-plate junctions can be described as

$$
\begin{gathered}
\Pi_{B}=V_{B}^{R}+V_{B}^{L} \\
\Pi_{C}=\sum_{r=1}^{N_{r}} V_{C}^{r}
\end{gathered}
$$

where, $V_{B}^{R}$ and $V_{B}^{L}$ represent the potential energy stored in the right and left side end boundaries of the cylindrical shells. And the $V_{C}^{r}$ indicates interface potential between the junctions of the $r$ th annular plate and cylindrical shells. They can be expressed as: 


$$
\begin{aligned}
& V_{B}^{R}=\left.\frac{1}{2} \int_{0}^{2 \pi}\left\{k_{x 1}^{R}\left(u_{s}^{1}\right)^{2}+k_{\theta 1}^{R}\left(v_{s}^{1}\right)^{2}+k_{r 1}^{R}\left(w_{s}^{1}\right)^{2}+K_{r 1}^{R}\left(\frac{\partial w_{s}^{1}}{\partial x}\right)^{2}\right\}\right|_{x=0, s=b} R_{o} \mathrm{~d} \theta \\
& +\left.\frac{1}{2} \int_{0}^{2 \pi}\left\{k_{x 1}^{R}\left(u_{s}^{2}\right)^{2}+k_{\theta 1}^{R}\left(v_{s}^{2}\right)^{2}+k_{r 1}^{R}\left(w_{s}^{2}\right)^{2}+K_{r 1}^{R}\left(\frac{\partial w_{s}^{2}}{\partial x}\right)^{2}\right\}\right|_{x=0, s=0} R_{i} \mathrm{~d} \theta \\
& V_{B}^{L}=\left.\frac{1}{2} \int_{0}^{2 \pi}\left\{k_{x 2}^{R}\left(u_{s}^{1}\right)^{2}+k_{\theta 2}^{R}\left(v_{s}^{1}\right)^{2}+k_{r 2}^{R}\left(w_{s}^{1}\right)^{2}+K_{r 2}^{R}\left(\frac{\partial w_{s}^{1}}{\partial x}\right)^{2}\right\}\right|_{x=L, s=b} R_{o} \mathrm{~d} \theta \\
& +\left.\frac{1}{2} \int_{0}^{2 \pi}\left\{k_{x 2}^{R}\left(u_{s}^{2}\right)^{2}+k_{\theta 2}^{R}\left(v_{s}^{2}\right)^{2}+k_{r 2}^{R}\left(w_{s}^{2}\right)^{2}+K_{r 2}^{R}\left(\frac{\partial w_{s}^{2}}{\partial x}\right)^{2}\right\}\right|_{x=L, s=0} R_{i} \mathrm{~d} \theta \\
& V_{C}^{r}=\left.\frac{1}{2} \int_{0}^{2 \pi}\left\{k_{x 1}^{c_{r}}\left(u_{s}^{1}-w_{p}^{r}\right)^{2}+k_{\theta 1}^{c_{r}}\left(v_{s}^{1}-v_{p}^{r}\right)^{2}+k_{r 1}^{c_{r}}\left(w_{s}^{1}-u_{p}^{r}\right)^{2}+K_{r 1}^{c_{r}}\left(\frac{\partial w_{s}^{1}}{\partial x}-\frac{\partial w_{p}^{r}}{\partial s}\right)^{2}\right\}\right|_{x=x_{c}, s=b} R_{o} \mathrm{~d} \theta \\
& +\left.\frac{1}{2} \int_{0}^{2 \pi}\left\{k_{x 2}^{c_{r}}\left(u_{s}^{2}-w_{p}^{r}\right)^{2}+k_{\theta 2}^{c_{r}}\left(v_{s}^{2}-v_{p}^{r}\right)^{2}+k_{r 2}^{c_{r}}\left(w_{s}^{2}-u_{p}^{r}\right)^{2}+K_{r 2}^{c_{r}}\left(\frac{\partial w_{s}^{2}}{\partial x}-\frac{\partial w_{p}^{r}}{\partial s}\right)^{2}\right\}\right|_{x=x_{c}, s=0} R_{i} \mathrm{~d} \theta
\end{aligned}
$$

\subsection{Admissible functions}

In order to derive the eigen equation of motion for the shell-plate combination, the displacements should be expanded in terms of generalized coordinates and admissible functions. The accuracy of the present work is critically dependent on the appropriate selections of the admissible functions. It should be noted that the boundary conditions are both relaxed and enforced by artificial springs, and there is no need to explicitly satisfy the continuity condition for the admissible functions. Considering of the circumferential symmetry of the structure, the displacements of annular palates and cylindrical shells, regardless of boundary conditions, can be invariantly sought as Fourier series expansions in addition to several supplementary functions expansions $[7,8]$

$$
u_{p}(s, \theta, t)=e^{j \omega t}\left(\begin{array}{l}
\sum_{m=0}^{\infty} \sum_{n=0}^{\infty} A_{m n}^{p} \cos \left(\lambda_{m p} s\right) \cos (n \theta)+\sum_{l=1}^{2} \sum_{n=0}^{\infty} \mathscr{A}_{l n}^{\theta} \xi_{l}(s) \cos (n \theta) \\
\sum_{m=0}^{\infty} \sum_{n=1}^{\infty} \bar{A}_{m n}^{p} \cos \left(\lambda_{m p} s\right) \sin (n \theta)+\sum_{l=1}^{2} \sum_{n=1}^{\infty} \overline{A_{l n}} \xi_{l}(s) \sin (n \theta)
\end{array}\right)
$$




$$
\begin{aligned}
& v_{p}(s, \theta, t)=e^{j \omega t}\left(\begin{array}{l}
\sum_{m=0}^{\infty} \sum_{n=1}^{\infty} B_{m n}^{p} \cos \left(\lambda_{m p} s\right) \sin (n \theta)+\sum_{l=1}^{2} \sum_{n=1}^{\infty} \beta_{l n}^{\infty} \xi_{l}(s) \sin (n \theta) \\
\sum_{m=0}^{\infty} \sum_{n=0}^{\infty} \bar{B}_{m n}^{p} \cos \left(\lambda_{m p} s\right) \cos (n \theta)+\sum_{l=1}^{2} \sum_{n=0}^{\infty} \bar{B}_{l n}^{c} \xi_{l}(s) \cos (n \theta)
\end{array}\right) \\
& w_{p}(s, \theta, t)=e^{j \omega t}\left(\begin{array}{l}
\sum_{m=0}^{\infty} \sum_{n=0}^{\infty} C_{m n}^{p} \cos \left(\lambda_{m p} s\right) \cos (n \theta)+\sum_{l=1}^{4} \sum_{n=0}^{\infty} \mathcal{C}_{l n}^{\infty} \zeta_{l}(s) \cos (n \theta) \\
\sum_{m=0}^{\infty} \sum_{n=1}^{\infty} \bar{C}_{m n}^{p} \cos \left(\lambda_{m p} s\right) \sin (n \theta)+\sum_{l=1}^{4} \sum_{n=1}^{\infty} \overline{\mathcal{E}}_{l n} \zeta_{l}(s) \sin (n \theta)
\end{array}\right) \\
& u_{s}(x, \theta, t)=e^{j \omega t}\left(\begin{array}{l}
\sum_{m=0}^{\infty} \sum_{n=0}^{\infty} A_{m n}^{s} \cos \left(\lambda_{m s} x\right) \cos (n \theta)+\sum_{l=1}^{2} \sum_{n=0}^{\infty} \mathscr{A}_{l n} \xi_{l}(x) \cos (n \theta) \\
\sum_{m=0}^{\infty} \sum_{n=1}^{\infty} \bar{A}_{m n}^{s} \cos \left(\lambda_{m p} x\right) \sin (n \theta)+\sum_{l=1}^{2} \sum_{n=1}^{\infty} \bar{A}_{l n}^{s} \xi_{l}(x) \sin (n \theta)
\end{array}\right) \\
& v_{s}(x, \theta, t)=e^{j \omega t}\left(\begin{array}{l}
\sum_{m=0}^{\infty} \sum_{n=1}^{\infty} B_{m n}^{s} \cos \left(\lambda_{m s} x\right) \sin (n \theta)+\sum_{l=1}^{2} \sum_{n=1}^{\infty} B_{\ln }^{\odot} \xi_{l}(x) \sin (n \theta) \\
\sum_{m=0}^{\infty} \sum_{n=0}^{\infty} \bar{B}_{m n}^{s} \cos \left(\lambda_{m s} x\right) \cos (n \theta)+\sum_{l=1}^{2} \sum_{n=0}^{\infty} \bar{B}_{l n}^{\odot} \xi_{l}(x) \cos (n \theta)
\end{array}\right) \\
& w_{s}(s, \theta, t)=e^{j \omega t}\left(\begin{array}{l}
\sum_{m=0}^{\infty} \sum_{n=0}^{\infty} C_{m n}^{s} \cos \left(\lambda_{m s} x\right) \cos (n \theta)+\sum_{l=1}^{4} \sum_{n=0}^{\infty} \mathcal{C}_{l n} \zeta_{l}(x) \cos (n \theta) \\
\sum_{m=0}^{\infty} \sum_{n=1}^{\infty} \bar{C}_{m n}^{s} \cos \left(\lambda_{m s} x\right) \sin (n \theta)+\sum_{l=1}^{4} \sum_{n=1}^{\infty} \overline{\mathcal{C}_{l n}} \zeta_{l}(x) \sin (n \theta)
\end{array}\right)
\end{aligned}
$$

where $\omega$ is the angular frequency, $t$ denotes time, $\lambda_{m p}=m \pi / b, \lambda_{m s}=m \pi / L$, non-negative integer $n$ are the circumferential wave number. $A_{m n}, \mathscr{A}_{l n}^{o}, \bar{A}_{m n}, \bar{A}_{l n}^{o}, B_{m n}, B_{l n}^{o}, \bar{B}_{m n}, \bar{B}_{l n}^{O}, C_{m n}, \mathcal{E}_{l n}^{o}$, $\bar{C}_{m n}, \overline{\mathcal{C}}_{l n}$ are the coefficients of the series which will be ascertained during the Rayleigh-Ritz procedure. $\xi_{l}(l=1,2)$ and $\zeta_{l}(l=1,2,3,4)$ are the supplementary functions for the in-plane and flexural displacements separately. The main reason of introducing the supplementary functions to two-dimensional Fourier series is to remove any potential discontinuities of displacement functions and their certain spatial derivatives on the boundaries of the structures. Moreover, the convergence rate and accuracy of the Fourier series will be enhanced by the supplementary functions. Theoretically, the functions $\xi_{l}$ and $\zeta_{l}$ can be represented as arbitrary continuous functions regardless of boundary 
conditions. However, the second order derivatives for the radial displacement and the first order derivatives for the in-plane displacements should be exist to ensure the continuity of displacements and its derivatives at any point on the double shells structure. Hence, both $\xi_{l}$ and $\zeta_{l}$ must be closed-form functions and sufficiently smooth throughout the whole coupled structure including the end boundaries and junctions. Such requirements can be satisfied by selecting simple polynomials as

$$
\begin{aligned}
& \xi_{1}(x)=x\left(\frac{x}{L_{x}}-1\right)^{2}, \quad \xi_{2}(x)=\frac{x^{2}}{L_{x}}\left(\frac{x}{L_{x}}-1\right) \\
& \zeta_{1}(x)=\frac{9 L_{x}}{4 \pi} \sin \left(\frac{\pi x}{2 L_{x}}\right)-\frac{L_{x}}{12 \pi} \sin \left(\frac{3 \pi x}{2 L_{x}}\right) \\
& \zeta_{2}(x)=-\frac{9 L_{x}}{4 \pi} \cos \left(\frac{\pi x}{2 L_{x}}\right)-\frac{L_{x}}{12 \pi} \cos \left(\frac{3 \pi x}{2 L_{x}}\right) \\
& \zeta_{3}(x)=\frac{L_{x}^{3}}{\pi^{3}} \sin \left(\frac{\pi x}{2 L_{x}}\right)-\frac{L_{x}^{3}}{3 \pi^{3}} \sin \left(\frac{3 \pi x}{2 L_{x}}\right) \\
& \zeta_{4}(x)=-\frac{L_{x}^{3}}{\pi^{3}} \cos \left(\frac{\pi x}{2 L_{x}}\right)-\frac{L_{x}^{3}}{3 \pi^{3}} \cos \left(\frac{3 \pi x}{2 L_{x}}\right)
\end{aligned}
$$

where the $L_{x}$ denotes the meridional length of the structure, with $L_{x}=b$ for the annular plates and $L_{x}=L$ for the cylindrical shells. It is easy to be proved that the supplementary functions are satisfied as

$$
\begin{aligned}
& \xi_{1}(0)=\xi_{1}(L)=\xi_{1}^{\prime}(L)=0, \quad \xi_{1}^{\prime}(0)=1 \\
& \xi_{2}(0)=\xi_{2}(L)=\xi_{2}^{\prime}(0)=0, \quad \xi_{2}^{\prime}(L)= \\
& \xi_{1}^{\prime}(0)=\xi_{3}^{\prime \prime \prime}(0)=\xi_{1}^{\prime}(L)=\xi_{4}^{\prime \prime \prime}(L)=1
\end{aligned}
$$




\subsection{Solution procedure}

In this paper, the Rayleigh-Ritz energy method is used to establish the equation of motion for the shell-plate coupling structure due to its flexibility and efficiency in practical calculation. The Lagrange functional for the coupled structure under vibration can be expressed as

$$
\Pi_{T o l}=\Pi_{T}+\Pi_{C}+\Pi_{B}+\Pi_{F}
$$

For the sake of brevity in prescribing the current solution procedure, only one annular plate is considered in the formulation. The current solution procedure can be used to derive the characteristic equation of the structure with more coupling annular plates readily. Substituting Eqs. (1), (18) and (37-38) into Eq. (59) together with the admissible functions and taking its derivatives with respect to each of the admissible function expansion coefficients, i.e.,

$$
\frac{\partial \prod_{T o l}}{\partial \alpha}=0, \quad \alpha=A_{m n}, \tilde{A}_{l n}, \bar{A}_{m n}, \overline{\tilde{A}}_{l n}, B_{m n}, \tilde{B}_{l n}, \bar{B}_{m n}, \overline{\tilde{B}}_{l n}, C_{m n}, \tilde{C}_{l n}, \bar{C}_{m n}, \overline{\tilde{C}}_{l n}
$$

Then the final system equation can be obtained and summarized in a matrix form as

$$
\left\{\mathbf{K}-\omega^{2} \mathbf{M}\right\} \mathbf{D}=\mathbf{F}
$$

where $\mathbf{K}$ and $\mathbf{M}$ are, respectively, the stiffness and mass matrices of the coupling structure. $\mathbf{D}$ and $\mathbf{F}$ represent the coefficient vector and external force vector. They are written as

$$
\begin{aligned}
\mathbf{K} & =\left[\begin{array}{ccc}
\mathbf{K}_{p p} & \mathbf{K}_{p s 1} & \mathbf{K}_{p s 2} \\
\mathbf{K}_{p s 1} \mathrm{~T} & \mathbf{K}_{s 1 s 1} & \mathbf{0} \\
\mathbf{K}_{p s 2}{ }_{\mathrm{T}}^{\mathrm{T}} & \mathbf{0} & \mathbf{K}_{s 2 s 2}
\end{array}\right], \quad \mathbf{M}=\left[\begin{array}{ccc}
\mathbf{M}_{p p} & & \\
& \mathbf{M}_{s 1 s 1} & \\
& & \mathbf{M}_{s 2 s 2}
\end{array}\right] \\
\mathbf{D} & =\left[\mathbf{A}_{p}, \mathbf{B}_{p}, \mathbf{C}_{p}, \mathbf{A}_{s 1}, \mathbf{B}_{s 1}, \mathbf{C}_{s 1}, \mathbf{A}_{s 2}, \mathbf{B}_{s 2}, \mathbf{C}_{s 2}\right]^{\mathrm{T}} \\
\mathbf{F} & =\left\{\begin{array}{lll}
\mathbf{0} & \mathbf{0} & \mathbf{F}_{s 2}
\end{array}\right\}^{\mathrm{T}}, \quad \mathbf{F}_{s 2}=\left\{\begin{array}{lll}
\mathbf{0} & \mathbf{0} & \mathbf{F}_{s 2, w}
\end{array}\right\}^{\mathrm{T}}
\end{aligned}
$$

where

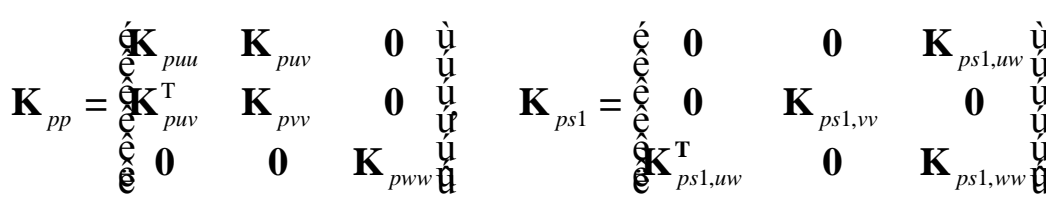




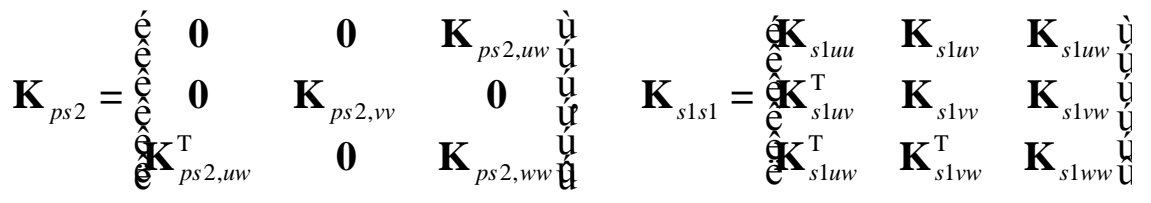

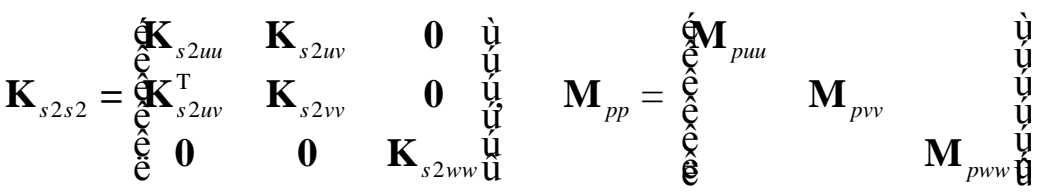

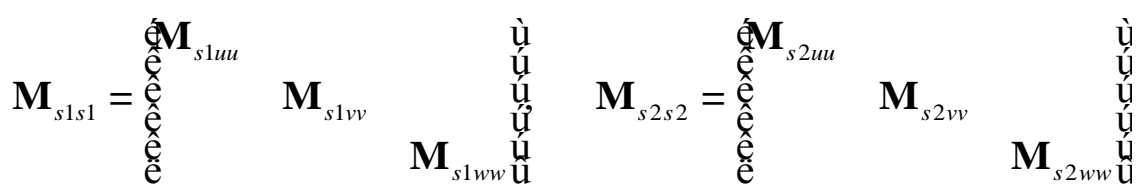

$$
\begin{aligned}
& \mathbf{A}_{p}=\left[A_{00}^{p}, \ldots, A_{m n}^{p}, \ldots, A_{M N}^{p}, \mathscr{A}_{10}^{\otimes}, \ldots, \mathscr{A}_{l n}^{\oplus}, \ldots, \mathscr{Q}_{2 N}^{\omega}, \bar{A}_{01}^{p}, \ldots, \bar{A}_{m n}^{p}, \ldots, \bar{A}_{M N}^{p}, \overline{\mathscr{A}}_{11}^{\omega}, \ldots, \overline{\mathscr{A}}_{l n}^{\omega}, \ldots, \overline{\mathscr{A}}_{2 N}^{\omega}\right] \\
& \mathbf{B}_{p}=\left[B_{01}^{p}, \ldots, B_{m n}^{p}, \ldots, B_{M N}^{p}, \beta_{11}^{\oplus}, \ldots, B_{l n}^{\Phi}, \ldots, B_{2 N}^{\oplus}, \bar{B}_{00}^{p}, \ldots, \bar{B}_{m n}^{p}, \ldots, \bar{B}_{M N}^{p}, \bar{B}_{10}^{\omega}, \ldots, \bar{B}_{l n}^{\omega}, \ldots, \bar{B}_{2 N}^{\omega}\right]
\end{aligned}
$$

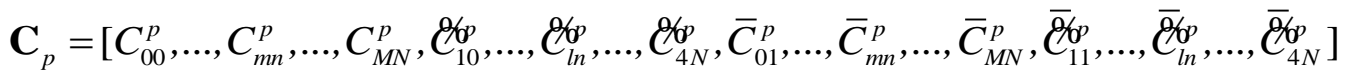

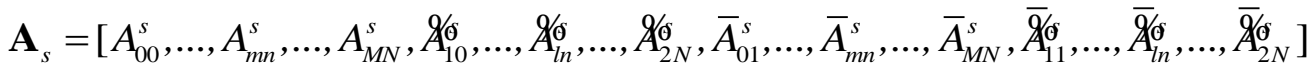

$$
\begin{aligned}
& \mathbf{B}_{s}=\left[B_{01}^{s}, \ldots, B_{m n}^{s}, \ldots, B_{M N}^{s}, B_{11}^{\odot}, \ldots, B_{l n}^{\odot}, \ldots, B_{2 N}^{\Theta}, \bar{B}_{00}^{s}, \ldots, \bar{B}_{m n}^{s}, \ldots, \bar{B}_{M N}^{s}, \bar{B}_{10}^{\odot}, \ldots, \bar{B}_{l n}^{\odot}, \ldots, \bar{B}_{2 N}^{*}\right]
\end{aligned}
$$

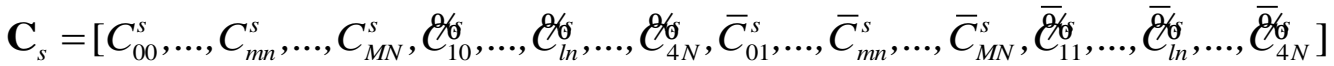

$M$ and $N$ are the truncation number of $m$ and $n$ respectively. The coefficient vectors $\mathbf{A}_{s 2}, \mathbf{B}_{s 2}$ and $\mathbf{C}_{s 2}$ are of the same form with $\mathbf{A}_{s 1}, \mathbf{B}_{s 1}$ and $\mathbf{C}_{s 1}$. In the matrixes, the subscript $s 1, s 2$ and $p$ represent the outer shell, the inner shell and the annular plate. Detailed expressions of sub-matrices in $\mathbf{K}$ and $\mathbf{M}$ are given in Appendix A.

Base on the Eq. (61), the coefficient vector can be directly obtained as

$$
\mathbf{X}=\left(\mathbf{K}-\omega^{2} \mathbf{M}\right)^{-1} \mathbf{F}
$$

It should be noted that, when excitations are imposed in circumferential and axial directions, the

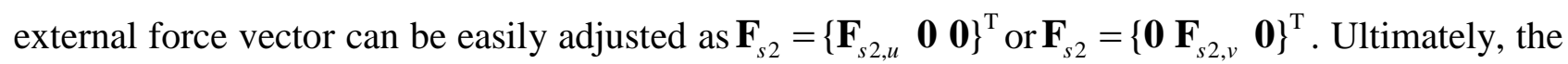
displacement functions of $u, v$ and $w$ can be obtained by substituting the coefficient vectors into Eqs. 
(42-47). Since the displacement functions are constructed smoothly, other variables like bending moments and shear forces can be obtained directly based on the displacement-strain relations.

Especially, for the free vibration analysis, the external force vector $\mathbf{F}$ in Eq. (61) should be replaced by null vector. Then the natural frequencies and eigenvectors of the coupled structure can be obtained by solving the eigen equation of Eq. (61). Subsequently, the mode shapes can be easily derived by substituting the coefficient vector $\mathbf{D}$ into the displacement expressions.

\section{Numerical examples and discussions}

With the theoretical formulation and solution procedure presented, several numerical examples are implemented in this part. Firstly, convergence and accuracy of the present method are validated. Then, the effects of elastic restraint and dimensional parameters on the free vibration characteristics of the coupled structure are investigated. The forced vibration characteristics of the double-shell structures are analyzed in the last subsection. In all examples, the material properties are selected as follows: $E_{p}=E_{s}=2.06 \times 10^{11} \mathrm{~N} / \mathrm{m}^{2}, \rho_{p}=\rho_{s}=7850 \mathrm{~kg} / \mathrm{m}^{3}, \mu_{p}=\mu_{s}=0.3$. The corresponding spring stiffnesses for three types of classical end boundaries are gave in Table 1. In addition, the zero natural frequency corresponding to the rigid-body mode of the coupled structure is neglected in following analysis.

\subsection{Convergence}

Theoretically, there are infinite terms of modified Fourier series. However, in the practical calculation the series should be numerically truncated and only finite terms are taken into consideration. Consequently, convergence study is of great importance in checking the convergence of the expansion series. Table 2 lists the fundamental natural frequencies of wave numbers $n=1-7$ for the double-shell structures under F-F, S-S and C-C end boundary conditions. In the table, the 
abbreviation E.B represents the end boundary condition. The geometry parameters used in the study are: $L=3 \mathrm{~m}, R_{o}=0.5 \mathrm{~m}, R_{i}=0.3 \mathrm{~m}$ and $h_{s}=h_{p}=0.005 \mathrm{~m}$. Assuming that all the coupling manners are close coupled, the stiffnesses of coupling boundary springs are set as infinite. The infinite stiffness is actually represented by a very large number i.e. $10^{13}$. In the model, the annular plate is coupled at the middle position in the meridional direction of the shells. Since the coupled structure is symmetrical in the circumferential direction and the circumferential expansion terms automatically satisfy the equations of motion and boundary conditions, only the meridional truncated number $M$ varies from 10 to 15 while the circumferential truncated number $N$ is fixed at $N=10$ in the convergence test. From the Table 2, one can see that quite small changes of the natural frequencies appear when $M$ varies from 10 to 15 regardless of boundary conditions. More accurate results can be obtained by further increasing the truncated number. To further validate the accuracy and reliability the present method, more results will be presented in the following section.

\subsection{Validation}

In this subsection, two examples of free vibration analysis of the double-shell structures are conducted to further confirm the validity of the present method. Under the framework, the annular plates between the shells can be arranged at arbitrary axial positions as shown in Fig 1. The dimensions of the shell-plate combinations are given as follows: $R_{o}=0.5 \mathrm{~m}, R_{i}=0.4 \mathrm{~m}, h_{s}=h_{p}=$ $0.005 \mathrm{~m}, L=1.2 \mathrm{~m}$. From the literature interview, no analytical solutions for such kind of coupled structures have been published. The theoretical results are validated by comparing the present dates with those obtained from ANSYS program. In the ANSYS program, element Shell 181 is employed for both the cylindrical shells and the annular plates with the element edge length equal to 0.01 .

In the first case, two annular plates are coupled to the double-shell structure, with one plate at the left ends of the shells and the other one at the middle of the shells along the $x$ direction, that is, $x_{1}$ 
$=0$ and $x_{2}=0.6 \mathrm{~m}$. The end boundary conditions for the left and right end of the structure are set as free and clamped separately. The comparison results are given in Table 3. It is clearly observed from table 3 that the present results agree closely with those obtained from the finite element method. The discrepancies between the two results are very small and all of them are less than $1 \%$ except the one for the $(m=1, n=1)$ mode.

In the second case, a double-shell structure coupled with three annular plates which are arranged at $x_{1}=0.1 \mathrm{~m}, x_{2}=0.6 \mathrm{~m}$ and $x_{3}=1.1 \mathrm{~m}$ is considered. The comparison results of the two methods are listed in Table 4, from which we can see that the natural frequencies of two method are in good agreement with each other and most of the errors are less than $1 \%$. Moreover, the increase of the axial wave number doesn't lead to significant numerical errors, which means that good accuracy can also be achieved for higher-order natural frequencies. For different end boundary conditions, the numerical errors have little difference, which means the present method is reliable for different end boundary conditions.

For illustrative purposes, several representative mode shapes for the outer shell under F-F, S-S and $\mathrm{C}-\mathrm{C}$ end boundary conditions are given in Fig. 3. It should be noted that, the number $n$ in the brackets $(m, n)$ represents the circumferential wave number, and $m$ represents the $m$ th model shape when the circumferential wave number is fixed at $n$. The vibration characteristics of the double-shell structure can be seen vividly in the figures.

From the present analysis results as well as those in subsection 3.1, it can be validated that the present method is capable of solving free vibration problems of double-shell structure coupled by annular plates regardless of end boundary conditions. 


\subsection{Effects of the axial position of the annular plate}

In this subsection, the effects of the axial positions of the annular plates on the natural frequencies of the double-shell structures will be investigated. The geometric parameters of the double-shell structure are: $L=3 \mathrm{~m}, R_{o}=0.5 \mathrm{~m}, R_{i}=0.3 \mathrm{~m}, h_{s}=h_{p}=0.005 \mathrm{~m}$. Under the framework, the axial position of the plate can be changed easily during the calculation procedure. Fig. 4 shows the variation of the lowest three natural frequencies as the axial position of the annular plate moves from 0 to $L$ along the meridianal direction. Regardless of end boundary conditions, the variety curves of all frequencies are symmetrical due to the symmetry of the whole structure. From the pictures it can be seen that, as the annular plate moves to the middle of the structure, the frequencies keep increasing. In other words, the rigidity of the double-shell structure increases as the plate moves close to the middle of the shells along axial direction. It is interesting noted that, the variety curves of the 3rd order natural frequency under free supported boundary condition and the 1 th order natural frequency under simply supported boundary condition are nearly flat. The mode shapes of the two natural frequencies are depicted in Fig. 5. It is clear in Fig. 5 that the vibration modes are both axial vibration, hence the axial vibration can be weakly restrained by annular plate.

\subsection{Effects of the coupling and end boundary conditions}

The general boundary conditions of the shells are implemented by introducing four groups of continuously distributed artificial springs at each end of the shells and the coupling junctions between the shell-plate components to simulate the boundary forces and displacements. Arbitrary end boundary conditions and varies coupling boundary conditions can be easily generated by transforming the artificial spring values at certain stiffnesses. Thus, the effects of the boundary springs on the vibration behavior of the coupled structure should be investigated. The geometric 
parameters of the double-shell structure are given as: $L=3 \mathrm{~m}, R_{o}=0.5 \mathrm{~m}, R_{i}=0.3 \mathrm{~m}, h_{s}=h_{p}=0.005$ $\mathrm{m}, x_{1}=\mathrm{L} / 2$.

\subsubsection{Effects of the coupling boundary conditions}

In this part, the effects on the natural frequencies of coupling boundary conditions are investigated. The control variate method is employed in the following procedures. With only one group of connective springs being changed and the other three groups fixed, we can learn that which group of the four connective springs $\left(k_{x}^{c}, k_{\theta}^{c}, k_{r}^{c}\right.$ and $\left.K_{r}^{c}\right)$ effects the natural frequencies most by comparison. During the calculating procedure, only one group of coupling spring are set as a relative smaller number $10^{5}$ to simulate a relatively weaker coupling condition in corresponding direction and the other three groups of connective springs are set as $10^{13}$ unchanged. The lowest five natural frequencies are listed in Table 4 under different end boundary conditions. For the C-C and S-S end boundary conditions, only in the case of $k_{x}^{c}=10^{5}$, the natural frequencies changes obviously. For the F-F end boundary condition, except the $k_{x}^{c}$, the other three groups of connective springs also have obvious effects on the natural frequencies. But the effect degree of the $k_{x}^{c}$ is the largest. From the coupling condition introduced in subsection 2.2, we can see that the $k_{x}^{c}$ connects the out-plane displacement of the annular plate to the cylindrical shells. Thus the out-plane vibration of the annular plate has the largest influence on the natural frequencies of the coupling system in low frequency range.

\subsubsection{Effects of the end boundary conditions}

In this part, the effects of general end boundary conditions on the natural frequencies of the double-shell structure will be investigated. The effects of end boundary springs in axial, rotational, rational and circumferential directions on the natural frequencies are investigated respectively. The

non-dimensional stiffnesses $\hat{k}_{x}, \hat{K}_{r}, \hat{k}_{r}$ and $\hat{k}_{\theta}$ are introduced here as the ratios of corresponding 
spring stiffness to the in-plane stiffness of the shells defined as $K\left(K=E h /\left(1-\mu^{2}\right)\right)$, respectively. For simplicity and generality in the analysis, we just pay attention to a simple case: one end of the shells is clamped and only one group of end boundary springs is exerted on the other end.

In Table 5, the first seven orders natural frequencies are listed corresponding to certain clamped-elastic end supported configurations. It can be seen that when the values of the nondimensional stiffnesses equal $10^{-6}$, all the four cases are of identical natural frequencies, because all the end boundary conditions are approximate to a C-F one. The $\hat{K}_{r}$ has the little effect on the natural frequencies, because when the $\hat{K}_{r}$ increases from $10^{-6}$ to $10^{4}$ the natural frequencies remain nearly unchanged. When the values of $\hat{k}_{x}, \hat{k}_{r}$ and $\hat{k}_{\theta}$ changing from $10^{-6}$ to $10^{4}$, all the corresponding natural frequencies change obviously. Moreover, the influences of the $\hat{k}_{r}$ and $\hat{k}_{\theta}$ on the natural frequencies are almost the same with each other. For further research for the influences of $\hat{k}_{r}$ and $\hat{k}_{\theta}$, Figs.6-7 separately show the variations of three lower and three higher modes frequencies of the coupled structure with the non-dimensional stiffenesses of $\hat{k}_{r}$ and $\hat{k}_{\theta}$ varying from $10^{-7}$ to $10^{4}$. For the lower modes ( $m=1, n=1,2,3)$, in Fig. 6 , it is clear that the frequencies increase rapidly as the nondimensional stiffenesses varies from $10^{-4}$ to $10^{0}$, whereas the frequencies keep stable beyond the stiffensses range. Let's define the stiffnesses range from $10^{-4}$ to $10^{0}$ as "sensitive range" of nondimensional spring stiffness. When the non-dimensional stiffenesses exceed $10^{0}$ the values of frequencies remain stable, because the boundary condition is almost clamped. Variations of natural frequencies for higher modes ( $m=8, n=8,9,10)$ are plotted in Fig. 7 , and we can see that the "sensitive range" of non-dimensional spring stiffness moves right and the frequencies variances are much smaller. Therefore, the natural frequencies are more sensitive to the elastic end boundary conditions in lower frequency range than in higher frequency range. Furthermore, the variety curves of the higher 
modes for the case of elastic end boundaries of $\hat{k}_{r}$ and $\hat{k}_{\theta}$ are coincide with that for the case of $\hat{k}_{\theta}$ in the "sensitive range".

\subsection{Forced vibration}

\subsubsection{Validation of the forced vibration}

In order to verify the capability of the present method for forced vibration analysis of doubleshell structures, several results are presented in this part. For simplicity, only clamped supported end boundary condition is considered in the following procedure. The same model in this section 3.2 is employed in this section. A vertical force $F=10 \mathrm{~N}$ is applied on the inner shell at $x_{F}=0.6 \mathrm{~m}$, and $\theta_{F}$ $=0$. The response points are located at $x_{e 1}=0.6 \mathrm{~m}$ and $x_{e 2}=0.35 \mathrm{~m}$ on the inner and outer shell with $\theta_{e}=0$. In following calculation, the truncated number $M$ and $N$ are set as 15 . The range of analysis frequency is from 300 to $1000 \mathrm{~Hz}$ and the corresponding frequency step is set as $2 \mathrm{~Hz}$. The out-plane vibration response curves of exciting and transfer points on the inner and outer shell obtained by the present method and FEM are depicted in Figs.8-11 separately. The FEM is performed in ANSYS program, in which element shell 181 is adopted, and two kind of mesh size are adopted: $0.02 \times 0.02$ $\mathrm{m}^{2}$ and $0.01 \times 0.01 \mathrm{~m}^{2}$. In the figures, the solid blue line indicates the present solution, the red dashed line and green dot dash line indicates the ANSYS solution with different mesh size, respectively. It is seen that, only in higher frequency range around $1000 \mathrm{~Hz}$, there is little difference between the resonant peaks of the two method. Clearly, as the mesh size varies from $0.02 \mathrm{~m}$ to $0.01 \mathrm{~m}$, the FEM results move closer to the present results. Consequently, the main reason of the errors may be attributed to the well-known drawback of the FEM which the FE model meets difficulties to deal with numerical computation in high frequencies when the mesh is not fine enough. On the whole, the response results of the two method agree well with each other, which confirm the accuracy of present method to conduct the forced vibration analysis. 


\subsubsection{Effects of the annular plate on the forced vibration}

According to the aforementioned study, it can be validated that the present method is reliable for the forced vibration analysis. In this part some parameter studies will be conducted based on present method. As known to all, the parameters of annular plate play an important role in dynamic behaviors of the double shells structure. In the following analysis, the effects of the thickness and width of the annular plate on the dynamic responses of the double shells structure will be investigated. The computational model, external excitation and boundary conditions are the same as those in last part. The response point is located at $x_{e}=0.35 \mathrm{~m}, \theta=\pi / 3$.

Figs. 12-14 display the dynamic responses in radial, circumferential and axial directions with the annular plate thickness varying from 0.003 to $0.005 \mathrm{~m}$, respectively. It is observed that, as the thickness increases, the positions of the resonant peaks move right in low frequency range for the reason that the thickness increase of the annular plate enhances the rigidity of the structure. However, it is interesting to observe that the positions of resonant peaks move left on the contrary in certain high frequency ranges. In general, the model frequency and model shape mainly depend on the values and distributions of the model mass and model stiffness. So, the reason for the phenomenon may lie in the fact that as the width of the annular plate increases the model mass and stiffness both increase, in such case, if the model mass increases faster than the model stiffness the resonance frequency will decrease. At the same time, it is seen that the magnitudes of the vibration responses are almost not effected by the thickness variation of the annular plate.

Figs. 15-17 show the dynamic responses in radial, circumferential and axial direction with annular plate width varies from 0.08 to 0.10 to $0.12 \mathrm{~m}$ respectively. As the increase of the space between the two cylindrical shells, the resonant peaks move left, but in the higher frequency range we don't find similar phenomenon. The reason have been given in the last paragraph. In low 
frequency range, the magnitudes of the displacements in radial and axial directions all increase when the width of the annular plate decreases which means that the more close the two shells are connected the more energy is transmitted to the outer shell. For the dynamic response in circumferential direction, the width of the annular plates only affect positions of the resonant peaks and have little influence of the response amplitude.

From the above analysis a conclusion can be made that the dimensions of the annular plates have significant effects the natural frequency of the double shell structure and have much less effects on the amplitudes of the dynamic responses on the outer shell. But for the reason of structure complexity, the influences of the annular plates between the double shell structures on the dynamic responses are not always consistent in frequency domain.

\section{Conclusions}

In this paper, a unified formulation is developed for the free and forced vibration analysis of circular cylindrical double-shell structure with general boundary conditions. Under current framework, the displacement components are expanded using Fourier series for both the cylindrical shells and annular plates regardless of boundary conditions. Several supplementary functions are added to the Fourier series to improve the accuracy and the convergence of Fourier series. Arbitrary boundary conditions at the coupling interfaces and end boundaries can be obtained by modifying the stiffnesses of the four groups of boundary springs introduced. Since the displacement field is constructed adequately smooth throughout the entire solution domain, an exact solution is obtained by using Rayleigh-Ritz procedure based on the energy function of the whole system. Numerical results regarding free and forced vibration analysis of the double-shell structure coupled with annular plates are conducted to check the convergence and accuracy of the present method. Then, a variety 
of extra vibration results for the double-shell structure with various boundary conditions are given, which may serve as benchmark results for validating new computational methods in the future. Figures are given to show the effects of dimensions of the annular plate on the forced vibration responses of the structures. The accurate results obtained by present method compared with those from ANSYS for the cylindrical shell-annular plate combination show the applicability of present method for complex shell-plate combinations.

The present method is efficient and stable to solve the free and forced vibration problems of cylindrical shell-annular plate coupled structures. In subsequent work, the method will be employed to solve the sound radiation problem of similar structures.

\section{Acknowledgement}

The authors gratefully acknowledge the financial support from the National Science Foundation of China (Nos.51175098 and 51279035) and the Fundamental Research Funds for the Central Universities (No. HEUCFQ1401).

\section{Appendix A.}

The components of stiffness and mass matrices in Eqs. (62-71) are, respectively, given by

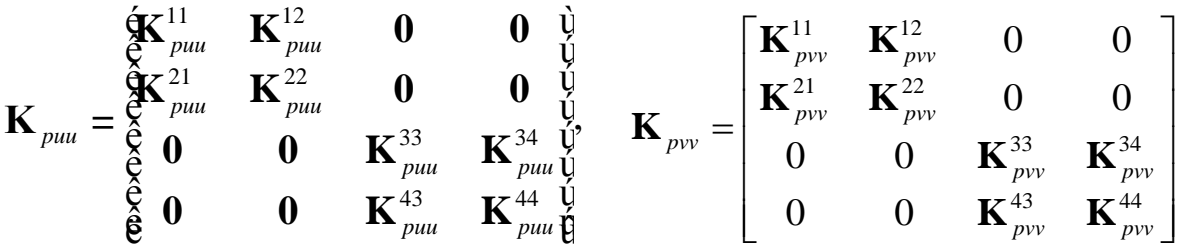

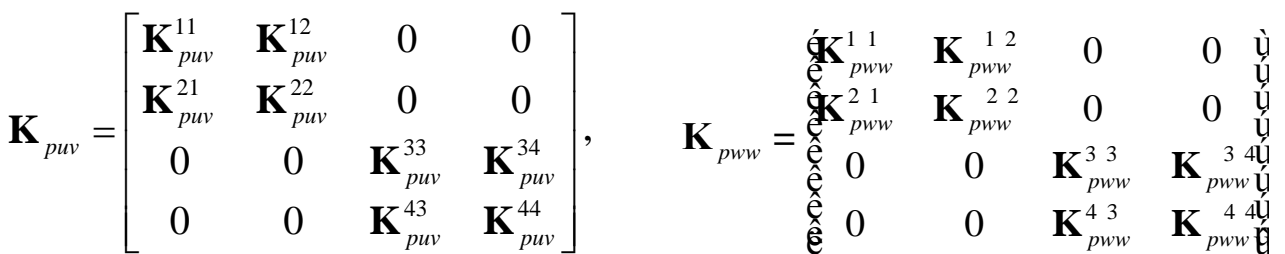




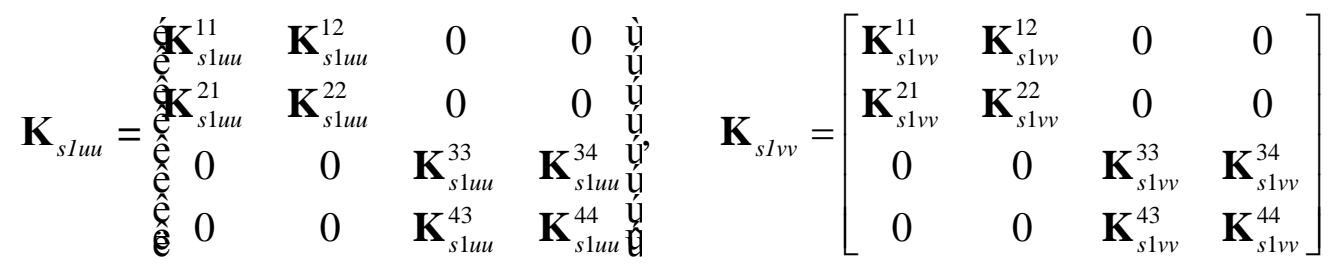

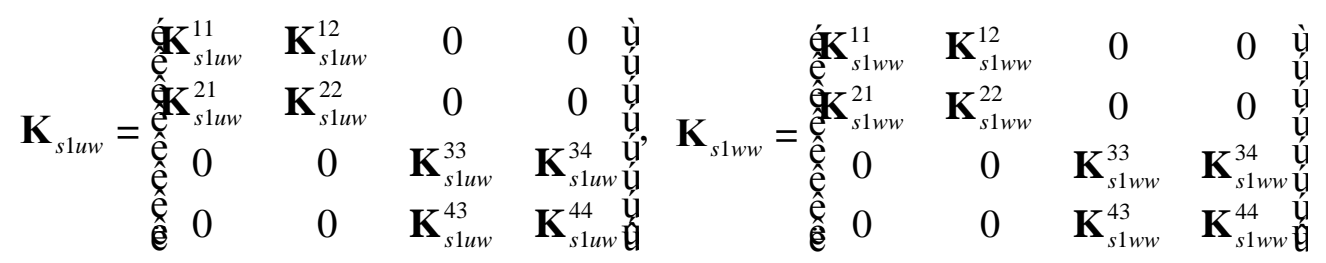

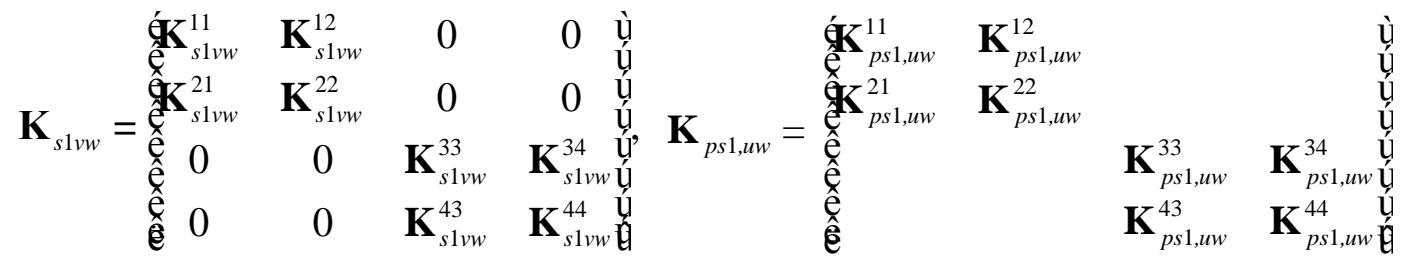

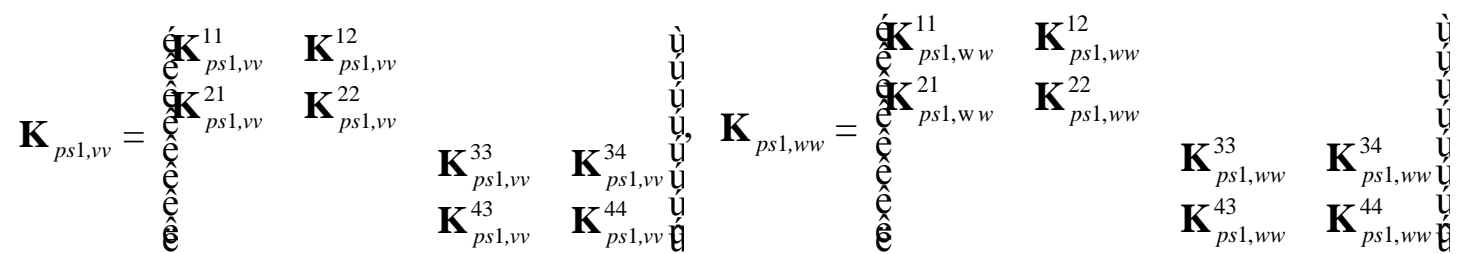

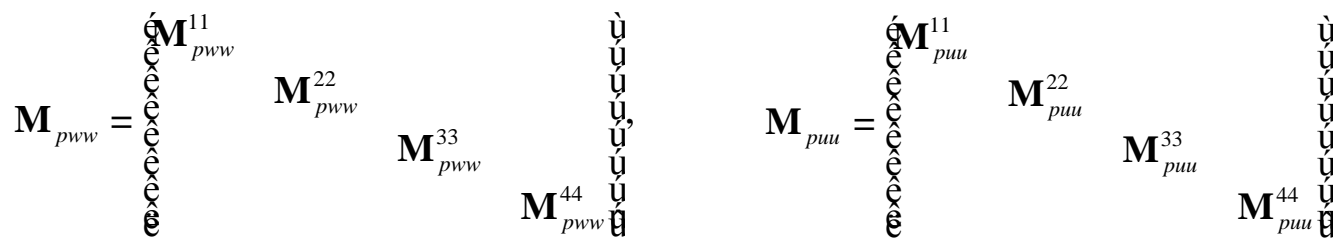

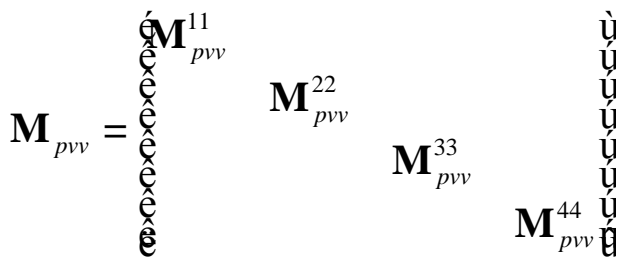

In the following annotations, only sub-matrixes with superscripts of $11,12,21$ and 22 are given, because the sub-matrixes with superscript of 33, 34, 43 and 44 are of the similar form.

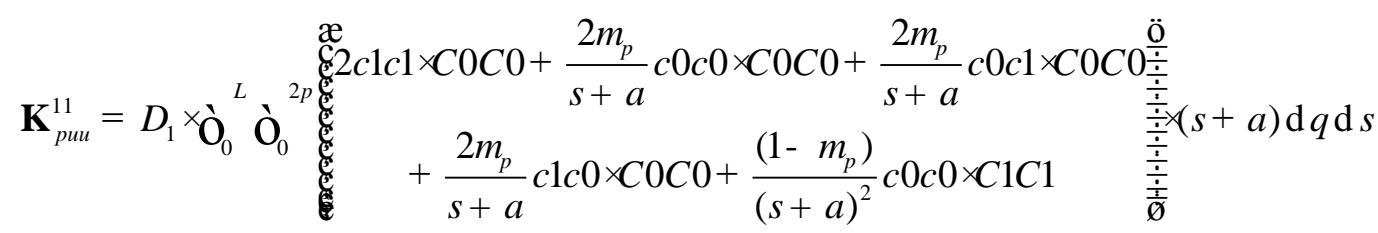




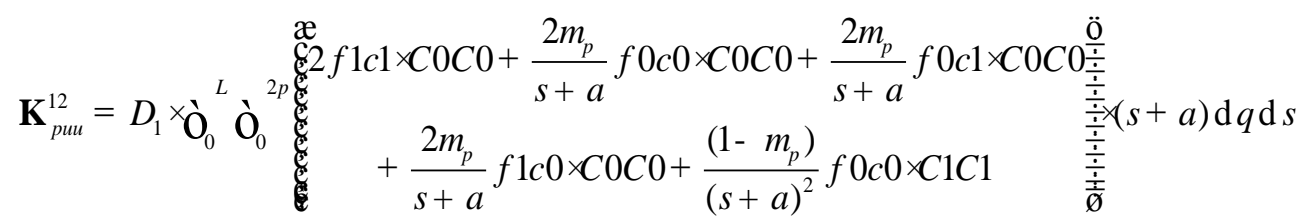

$$
\begin{aligned}
& \mathbf{K}_{p \text { рuи }}^{21}=\mathbf{K}_{\text {puu }}^{12}
\end{aligned}
$$

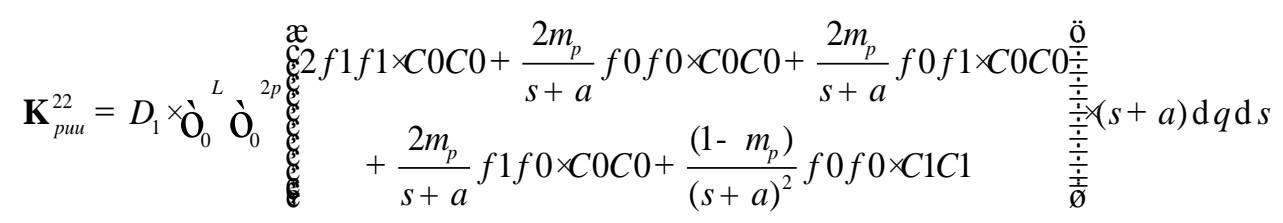

$$
\begin{aligned}
& \mathbf{K}_{p u v}^{11}=D_{1} \cdot \int_{0}^{b} \int_{0}^{2 \pi}\left(\begin{array}{l}
\frac{2}{(s+a)^{2}} c 0 c 0 \cdot C 0 S 1+\frac{2 \mu_{p}}{s+a} c 1 c 0 \cdot C 0 S 1 \\
+\frac{1-\mu_{p}}{s+a} c 0 c 1 \cdot C 1 S 0-\frac{1-\mu_{p}}{(s+a)^{2}} c 0 c 0 \cdot C 1 S 0
\end{array}\right) \cdot(s+a) \mathrm{d} \theta \mathrm{d} s \\
& \mathbf{K}_{p u v}^{12}=D_{1} \cdot \int_{0}^{b} \int_{0}^{2 \pi}\left(\begin{array}{l}
\frac{2}{(s+a)^{2}} f 0 c 0 \cdot C 0 S 1+\frac{2 \mu_{p}}{s+a} f 1 c 0 \cdot C 0 S 1 \\
+\frac{1-\mu_{p}}{s+a} f 0 c 1 \cdot C 1 S 0-\frac{1-\mu_{p}}{(s+a)^{2}} f 0 c 0 \cdot C 1 S 0
\end{array}\right) \cdot(s+a) \mathrm{d} \theta \mathrm{d} s \\
& \mathbf{K}_{p u v}^{21}=D_{1} \cdot \int_{0}^{b} \int_{0}^{2 \pi}\left(\begin{array}{c}
\frac{2}{(s+a)^{2}} f 0 c 0 \cdot C 0 S 1+\frac{2 \mu_{p}}{s+a} f 1 c 0 \cdot C 0 S 1 \\
+\frac{1-\mu_{p}}{s+a} f 0 c 1 \cdot C 1 S 0-\frac{1-\mu_{p}}{(s+a)^{2}} f 0 c 0 \cdot C 1 S 0
\end{array}\right) \cdot(s+a) \mathrm{d} \theta \mathrm{d} s \\
& \mathbf{K}_{p u v}^{22}=D_{1} \cdot \int_{0}^{b} \int_{0}^{2 \pi}\left(\begin{array}{l}
\frac{2}{(s+a)^{2}} f 0 f 0 \cdot C 0 S 1+\frac{2 \mu_{p}}{s+a} f 1 f 0 \cdot C 0 S 1 \\
+\frac{1-\mu_{p}}{s+a} f 0 f 1 \cdot C 1 S 0-\frac{1-\mu_{p}}{(s+a)^{2}} f 0 f 0 \cdot C 1 S 0
\end{array}\right) \cdot(s+a) \mathrm{d} \theta \mathrm{d} s \\
& \mathbf{K}_{p v v}^{11}=D_{1} \cdot \int_{0}^{b} \int_{0}^{2 \pi}\left(\begin{array}{c}
\frac{2}{(s+a)^{2}} c 0 c 0 \cdot S 1 S 1+\left(1-\mu_{p}\right) c 1 c 1 \cdot S 0 S 0-\frac{1-\mu_{p}}{s+a} c 1 c 0 \cdot S 0 S 0 \\
-\frac{1-\mu_{p}}{s+a} c 0 c 1 \cdot S 0 S 0+\frac{1-\mu_{p}}{(s+a)^{2}} c 0 c 0 \cdot S 0 S 0
\end{array}\right) \cdot(s+a) \mathrm{d} \theta \mathrm{d} s \\
& +\left.k_{\theta 1}^{c} \cdot R_{o} \cdot c 0 c 0\right|_{s=b} \cdot \int_{0}^{2 \pi} S 0 S 0 \mathrm{~d} \theta+\left.k_{\theta 2}^{c} \cdot R_{i} \cdot c 0 c 0\right|_{s=0} \cdot \int_{0}^{2 \pi} S 0 S 0 \mathrm{~d} \theta
\end{aligned}
$$




$$
\begin{aligned}
& \mathbf{K}_{p v v}^{12}=D_{1} \cdot \int_{0}^{b} \int_{0}^{2 \pi}\left(\begin{array}{c}
\frac{2}{(s+a)^{2}} f 0 c 0 \cdot S 1 S 1+\left(1-\mu_{p}\right) \mathrm{f} 1 c 1 \cdot S 0 S 0-\frac{1-\mu_{p}}{s+a} f 1 c 0 \cdot S 0 S 0 \\
-\frac{1-\mu_{p}}{s+a} f 0 c 1 \cdot S 0 S 0+\frac{1-\mu_{p}}{(s+a)^{2}} f 0 c 0 \cdot S 0 S 0
\end{array}\right) \cdot(s+a) \mathrm{d} \theta \mathrm{d} s \\
& +\left.k_{\theta 1}^{c} \cdot R_{o} \cdot c 0 c 0\right|_{s=b} \cdot \int_{0}^{2 \pi} S 0 S 0 \mathrm{~d} \theta+\left.k_{\theta 1} \cdot R_{i} \cdot c 0 c 0\right|_{s=0} \cdot \int_{0}^{2 \pi} S 0 S 0 \mathrm{~d} \theta \\
& \mathbf{K}_{p v v}^{21}=D_{1} \cdot \int_{0}^{b} \int_{0}^{2 \pi}\left(\begin{array}{c}
\frac{2}{(s+a)^{2}} c 0 f 0 \cdot S 1 S 1+\left(1-\mu_{p}\right) c 1 f 1 \cdot S 0 S 0-\frac{1-\mu_{p}}{s+a} c 1 f 0 \cdot S 0 S 0 \\
-\frac{1-\mu_{p}}{s+a} c 0 f 1 \cdot S 0 S 0+\frac{1-\mu_{p}}{(s+a)^{2}} c 0 f 0 \cdot S 0 S 0
\end{array}\right) \cdot(s+a) \mathrm{d} \theta \mathrm{d} s \\
& +\left.k_{\theta 1}^{c} \cdot R_{o} \cdot c 0 f 0\right|_{s=b} \cdot \int_{0}^{2 \pi} S 0 S 0 \mathrm{~d} \theta+\left.k_{\theta 2}^{c} \cdot R_{i} \cdot c 0 f 0\right|_{s=0} \cdot \int_{0}^{2 \pi} S 0 S 0 \mathrm{~d} \theta \\
& \mathbf{K}_{p v v}^{22}=D_{1} \cdot \int_{0}^{b} \int_{0}^{2 \pi}\left(\begin{array}{c}
\frac{2}{(s+a)^{2}} f 0 f 0 \cdot S 1 S 1+\left(1-\mu_{p}\right) f 1 f 1 \cdot S 0 S 0-\frac{1-\mu_{p}}{s+a} f 1 f 0 \cdot S 0 S 0 \\
-\frac{1-\mu_{p}}{s+a} f 0 f 1 \cdot S 0 S 0+\frac{1-\mu_{p}}{(s+a)^{2}} f 0 f 0 \cdot S 0 S 0
\end{array}\right) \cdot(s+a) \mathrm{d} \theta \mathrm{d} s \\
& +\left.k_{\theta 1}^{c} \cdot R_{o} \cdot c 0 f 0\right|_{s=b} \cdot \int_{0}^{2 \pi} S 0 S 0 \mathrm{~d} \theta+\left.k_{\theta 2}^{c} \cdot R_{i} \cdot c 0 f 0\right|_{s=0} \cdot \int_{0}^{2 \pi} S 0 S 0 \mathrm{~d} \theta
\end{aligned}
$$

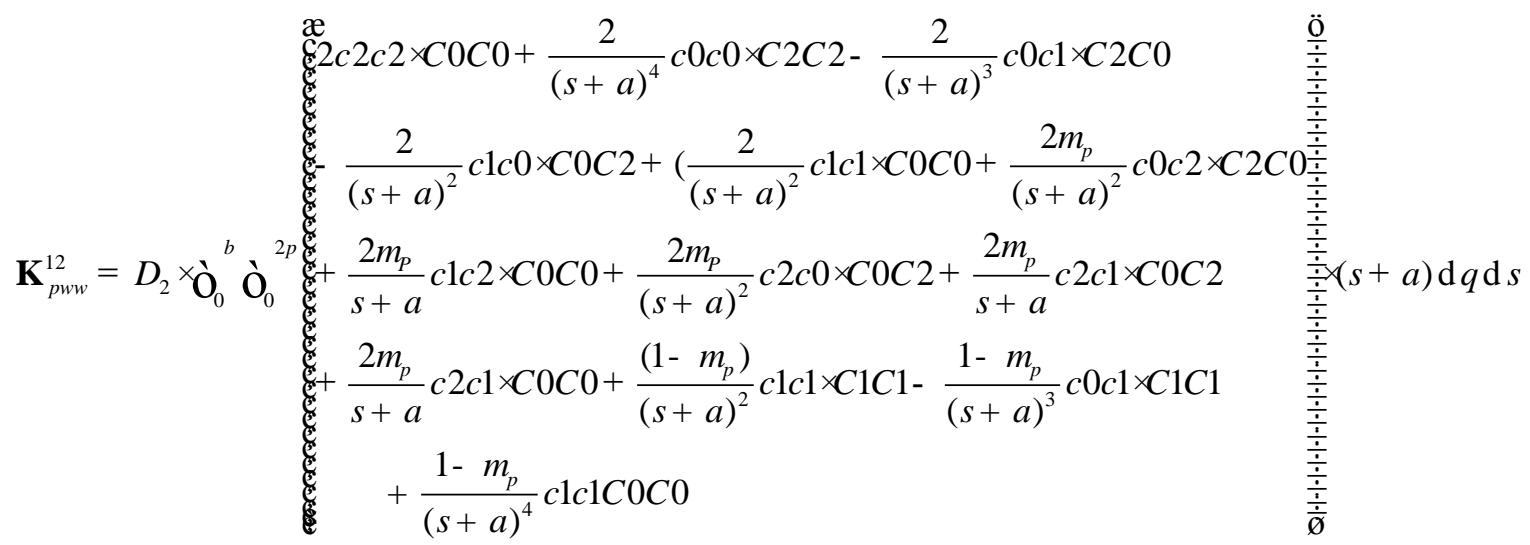

$$
\begin{aligned}
& +k_{x 1}^{c} \times R_{o} \times\left. c 0 c 0\right|_{s=b} \times \grave{\mathbf{O}}_{0}^{2 p} C 0 C 0 d q+k_{x 2}^{c} \times R_{i} \times\left. c 0 c 0\right|_{s=0} \times \grave{\mathbf{O}}_{0}^{2 p} C 0 C 0 \mathrm{~d} q \\
& +k_{x 1}^{c} \times R_{o} \times\left. c 1 c 1\right|_{s=b} \times \grave{\mathbf{O}}_{0}{ }^{2 p} C 0 C 0 d q+k_{x 2}^{c} \times R_{i} \times c 1 c 1_{s=0} \times \grave{\mathbf{O}}_{0}{ }^{2 p} C 0 C 0 \mathrm{~d} q
\end{aligned}
$$




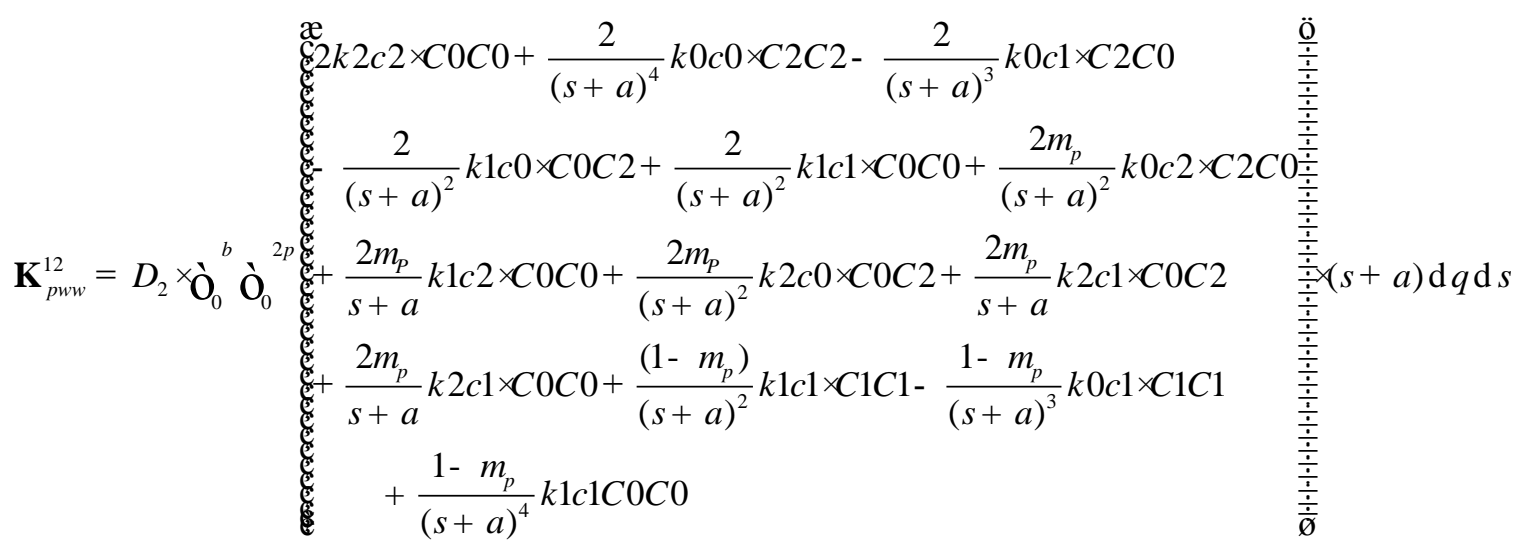

$$
\begin{aligned}
& +k_{s 1}^{c} \times R_{o} \times\left. k 0 c 0\right|_{s=b} \times \grave{\mathrm{O}}_{0}^{2 p} C 0 C 0 \mathrm{~d} q+k_{s 2}^{c} \times R_{i} \times\left. k 0 c 0\right|_{s=0} \times \grave{\mathbf{O}}_{0}^{2 p} C 0 C 0 \mathrm{~d} q \\
& +k_{s 1}^{c} \times R_{o} \times\left. k 1 c 1\right|_{s=b} \times \grave{\mathrm{O}}_{0}{ }^{2 p} C 0 C 0 \mathrm{~d} q+k_{x 2}^{c} \times R_{i} \times\left. k 1 c 1\right|_{s=0} \times \grave{\mathrm{O}}_{0}{ }^{2 p} C 0 C 0 \mathrm{~d} q \\
& \mathbf{K}_{p w w}^{12}=\mathbf{K}_{p w w}^{21}
\end{aligned}
$$

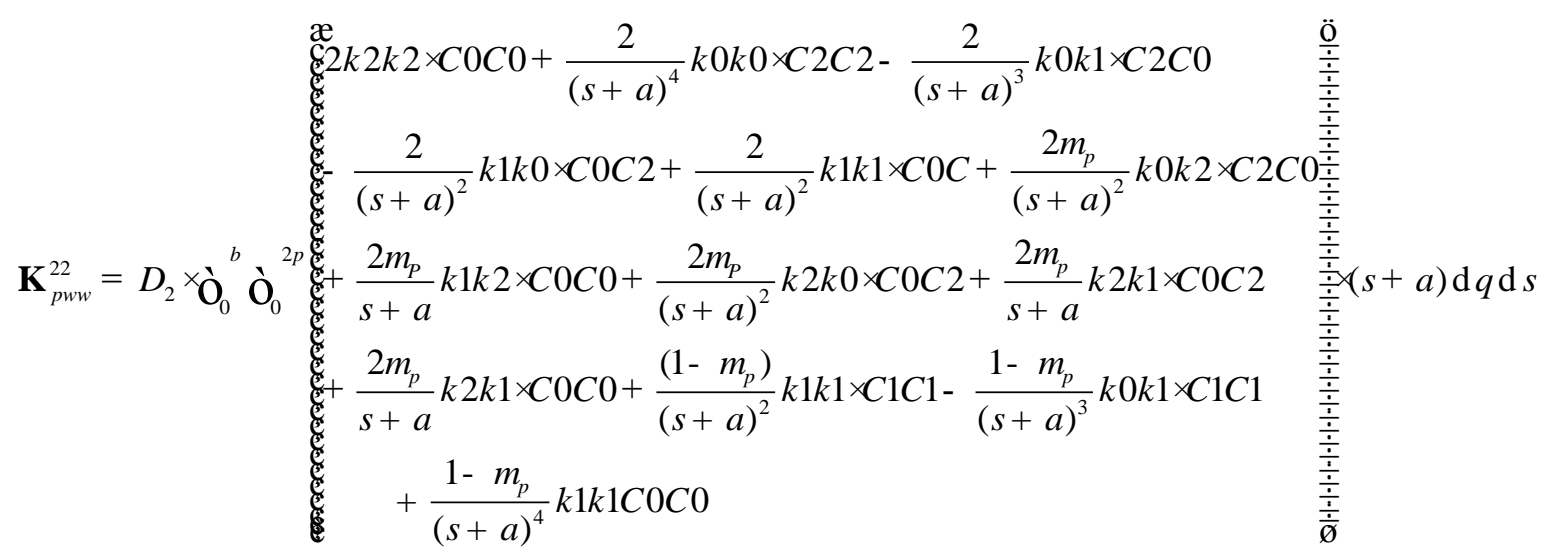

$$
\begin{aligned}
& +k_{s 1}^{c} \times R_{o} \times\left. k 0 k 0\right|_{s=b} \times \grave{\mathbf{O}}_{0}^{2 p} C 0 C 0 \mathrm{~d} q+k_{s 2}^{c} \times R_{i} \times\left. k 0 k 0\right|_{s=0} \times \grave{\mathbf{O}}_{0}^{2 p} C 0 C 0 \mathrm{~d} q \\
& +k_{s 1}^{c} \times R_{o} \times\left. k 1 k 1\right|_{s=b} \times \grave{\mathrm{O}}_{0}^{2 p} C 0 C 0 \mathrm{~d} q+k_{s 2}^{c} \times R_{i} \times\left. k 1 k 1\right|_{s=0} \times \grave{\mathrm{O}}_{0}^{2 p} C 0 C 0 \mathrm{~d} q
\end{aligned}
$$

$$
\begin{aligned}
& +k_{x 1}^{L} \times R_{o} \times\left. f 0 c 0\right|_{x=0} \grave{\mathbf{O}}_{0}^{2 p} C 0 C 0 \mathrm{~d} q+k_{x 1}^{R} \times R_{o} \times\left. f 0 c 0\right|_{x=L} \grave{\mathbf{O}}_{0}^{2 p} C 0 C 0 \mathrm{~d} q \\
& +k_{x 1}^{c} \times R_{o} \times\left. f 0 c 0\right|_{x=x c} \grave{\mathbf{O}}_{0}^{2 p} C 0 C 0 \mathrm{~d} q
\end{aligned}
$$




$$
\mathbf{K}_{\text {sluu }}^{21}=\mathbf{K}_{\text {sluu }}^{12}
$$

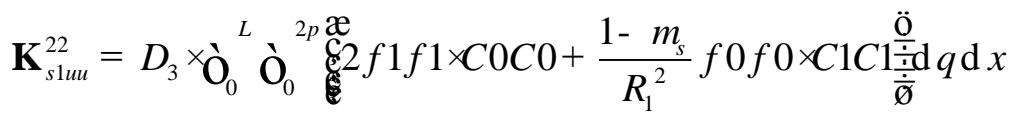

$$
\begin{aligned}
& +k_{x 1}^{L} \times R_{o} \times\left. f 0 f 0\right|_{x=0} \times \grave{\mathbf{O}}_{0}^{2 p} C 0 C 0 \mathrm{~d} q+k_{x 1}^{R} \times R_{o} \times\left. f 0 f 0\right|_{x=L} \grave{\mathbf{O}}_{0}^{2 p} C 0 C 0 \mathrm{~d} q \\
& +k_{x 1}^{c} \times R_{o} \times\left. f 0 f 0\right|_{x=x c} \times \grave{\mathbf{O}}_{0}^{2 p} C 0 C 0 \mathrm{~d} q
\end{aligned}
$$$$
\left.\mathbf{K}_{s 1 v v}^{11}=\int_{0}^{b} \int_{0}^{2 \pi}\left(\frac{2 D_{3}}{R_{2}}+\frac{2 D_{4}}{R_{2}}\right) \cdot c 0 c 0 \cdot S 1 S 1+\left(D_{3}+4 D_{4}\right) \cdot\left(1-\mu_{s}\right) \cdot c 1 c 1 \cdot S 0 S 0\right) \mathrm{d} \theta \mathrm{d} x
$$$$
+R_{o} \cdot\left(\left.k_{\theta 1}^{R} \cdot c 0 c 0\right|_{x=0}+\left.k_{\theta 1, s}^{L} \cdot c 0 c 0\right|_{x=L}+\left.k_{\theta 1}^{c} \cdot c 0 c 0\right|_{x=x_{c}}\right) \cdot \int_{0}^{2 \pi} \operatorname{SOS} 0 \mathrm{~d} \theta
$$

$$
\begin{gathered}
\left.\mathbf{K}_{s l v v}^{12}=\int_{0}^{b} \int_{0}^{2 \pi}\left(\frac{2 D_{3}}{R_{2}}+\frac{2 D_{5}}{R_{2}}\right) f 0 c 0 \cdot S 1 S 1+\left(D_{3}+4 D_{5}\right)\left(1-\mu_{s}\right) f 1 c 1 \cdot S 0 S 0\right) \mathrm{d} \theta \mathrm{d} x \\
+R_{1}\left(\left.k_{\theta 1}^{L} f 0 c 0\right|_{x=0}+\left.k_{\theta 1}^{R} f 0 c 0\right|_{x=L}+\left.k_{\theta 1}^{c} f 0 c 0\right|_{x=x_{c}}\right) \int_{0}^{2 \pi} S 0 S 0 \mathrm{~d} \theta
\end{gathered}
$$

$$
\begin{gathered}
\left.\mathbf{K}_{s l v v}^{21}=\int_{0}^{b} \int_{0}^{2 \pi}\left(\frac{2 D_{3}}{R_{2}}+\frac{2 D_{4}}{R_{2}}\right) c 0 f 0 \cdot S 1 S 1+\left(D_{3}+4 D_{4}\right)\left(1-\mu_{s}\right) c 1 f 1 \cdot S 0 S 0\right) \mathrm{d} \theta \mathrm{d} x \\
+R_{o}\left(\left.k_{\theta 1}^{L} f 0 c 0\right|_{x=0}+\left.k_{\theta 1}^{R} f 0 c 0\right|_{x=L}+\left.k_{\theta 1}^{c} f 0 c 0\right|_{x=x_{c}}\right) \int_{0}^{2 \pi} S 0 S 0 \mathrm{~d} \theta
\end{gathered}
$$

$$
\begin{gathered}
\left.\mathbf{K}_{s l v v}^{22}=\int_{0}^{b} \int_{0}^{2 \pi}\left(\frac{2 D_{3}}{R_{2}}+\frac{2 D_{4}}{R_{2}}\right) \cdot f 0 f 0 \cdot S 1 S 1+\left(D_{3}+4 D_{4}\right) \cdot\left(1-\mu_{s}\right) \cdot f 1 f 1 \cdot S 0 S 0\right) \mathrm{d} \theta \mathrm{d} x \\
+R_{o} \cdot\left(\left.k_{\theta 1}^{L} f 0 f 0\right|_{x=0}+\left.k_{\theta 1}^{R} f 0 c 0\right|_{x=L}+\left.k_{\theta 1}^{c} f 0 f 0\right|_{x=x_{c}}\right) \cdot \int_{0}^{2 \pi} S 0 S 0 \mathrm{~d} \theta
\end{gathered}
$$

$$
\mathbf{K}_{\text {sluw }}^{11}=D_{3} \times \grave{\mathbf{O}}_{0}^{L} \grave{\mathrm{O}}_{2}^{2 p} 2 R_{0} m_{s} c 1 c 0 \times C 0 C 0 \mathrm{~d} q \mathrm{~d} x
$$$$
\mathbf{K}_{\text {sluw }}^{12}=D_{3} \grave{\mathrm{O}}_{0}{ }^{L} \grave{\mathrm{O}}_{2}{ }^{2 p} 2 R_{0} m_{s} f 1 c 0 \times C 0 C 0 \mathrm{~d} q \mathrm{~d} x
$$$$
\mathbf{K}_{\text {sluw }}^{21}=D_{3} \times \grave{\mathbf{O}}_{0}{ }^{L} \grave{\mathbf{O}}_{2}{ }^{2 p} 2 R_{0} m_{s} c 1 k 0 \times C 0 C 0 \mathrm{~d} q \mathrm{~d} x
$$

$$
\mathbf{K}_{\text {sluw }}^{22}=D_{3} \times \grave{\mathrm{O}}_{0}^{L} \grave{\mathrm{O}}_{2}^{2 p} 2 R_{0} m_{s} f 1 k 0 \times C 0 C 0 \mathrm{~d} q \mathrm{~d} x
$$

$$
\mathbf{K}_{s 11 w}^{11}=D_{3} \times \grave{\mathrm{O}}_{0}^{L} \grave{\mathrm{O}}_{2}^{2 p} 2 / R_{0}^{2} \times m_{s} c 0 c 0 \times \operatorname{CoS} 1 \mathrm{~d} x \mathrm{~d} q
$$

$$
+D_{5} \times \grave{\mathrm{O}}_{0}^{L} \grave{\mathrm{O}}_{2}{ }^{2 p}-2 m_{s} c 2 c 0 \times C 0 S 1-2 / R_{0}^{2} \times c 0 c 0 \times C 2 S 1-4\left(1-m_{s}\right) c 1 c 1 \times C 1 S 0 \mathrm{~d} x \mathrm{~d} q
$$




$$
\begin{aligned}
\mathbf{K}_{s 1 v w}^{12}= & D_{3} \times \grave{\mathrm{O}}_{0}{ }^{L} \grave{\mathrm{O}}_{2}{ }_{2}^{2 p} \frac{2}{R_{0}^{2}} m_{s} c 0 k 0 \times C 0 S 1 \mathrm{~d} x \mathrm{~d} q \\
& +D_{5} \times \grave{\mathrm{O}}_{0}^{L} \grave{\mathrm{O}}_{2}^{2 p}-2 m_{s} c 2 k 0 \times C 0 S 1-\frac{2}{R_{0}^{2}} c 0 k 0 \times C 2 S 1-4\left(1-m_{s}\right) c 1 k 1 \times C 1 S 0 \mathrm{~d} x \mathrm{~d} q
\end{aligned}
$$

$$
\begin{aligned}
\mathbf{K}_{s l v w}^{21}= & D_{3} \times \grave{\mathrm{O}}_{0}^{L} \grave{\mathrm{O}}_{2}^{2 p} \frac{2}{R_{0}^{2}} m_{s} f 0 c 0 \times C 0 S 1 \mathrm{~d} x \mathrm{~d} q \\
& +D_{5} \grave{\mathrm{O}}_{0}^{L} \grave{\mathrm{O}}_{2}^{2 p}-2 m_{s} f 2 c 0 \times C 0 S 1-\frac{2}{R_{0}^{2}} f 0 c 0 \times C 2 S 1-4\left(1-m_{s}\right) f 1 c 1 \times C 1 S 0 \mathrm{~d} x \mathrm{~d} q
\end{aligned}
$$

$$
\begin{aligned}
\mathbf{K}_{s l v w}^{22}= & D_{3} \times \grave{\mathbf{O}}_{0}^{L} \grave{\mathrm{O}}_{2}^{2 p} \frac{2}{R_{0}^{2}} m_{s} f 0 k 0 \times C 0 S 1 \mathrm{~d} x \mathrm{~d} q \\
& +D_{5} \times \grave{\mathrm{O}}_{0}^{L} \grave{\mathrm{O}}_{2}^{2 p}-2 m_{s} f 2 k 0 \times C 0 S 1-\frac{2}{R_{0}^{2}} f 0 k 0 \times C 2 S 1-4\left(1-m_{s}\right) f 1 k 1 \times C 1 S 0 \mathrm{~d} x \mathrm{~d} q
\end{aligned}
$$

$$
\begin{aligned}
& \mathbf{K}_{s 1 w w}^{11}=\grave{\mathrm{O}}_{0}^{L} \grave{\mathrm{O}}_{0}^{2 p} \frac{2 D_{3}}{R_{o}{ }^{2}} c 0 c 0 \times C 0 C 0 \times R_{1} \mathrm{~d} x \mathrm{~d} q
\end{aligned}
$$

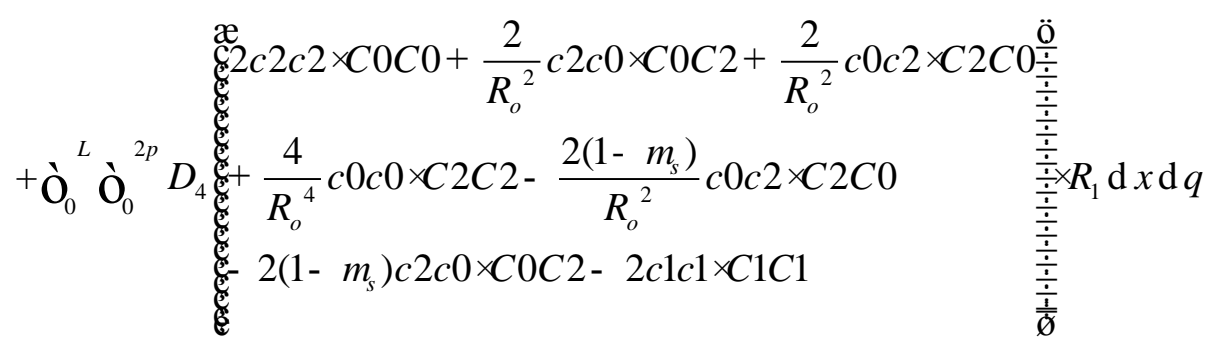

$$
\begin{aligned}
& +\left(\left.k_{r 1}^{L} x_{C} 0 c 0\right|_{x=0} \times C 0 C 0+K_{r 1}^{L} \times\left. c 1 c 1\right|_{x=0} \times C 0 C 0\right) \times R_{o} \\
& +\left(k_{r 1}^{R} \times\left. c 0 c 0\right|_{x=L} \times C 0 C 0+K_{r 1}^{R} \times\left. c 1 c 1\right|_{x=L} \times C 0 C 0\right) \times R_{o} \\
& +\left(k_{x 1}^{L} \times\left. c 0 c 0\right|_{x=0} \times C 0 C 0+k_{x 1}^{R} \times\left. c 1 c 1\right|_{x=L} \times C 0 C 0\right) \times R_{o} \\
& \mathbf{K}_{s 1 w w}^{12}=\grave{\mathrm{O}}_{0}^{L} \grave{\mathrm{O}}_{0}^{2 p} \frac{2 D_{3}}{R^{2}} k 0 c 0 \times C 0 C 0 \times R_{o} \mathrm{~d} x \mathrm{~d} q
\end{aligned}
$$

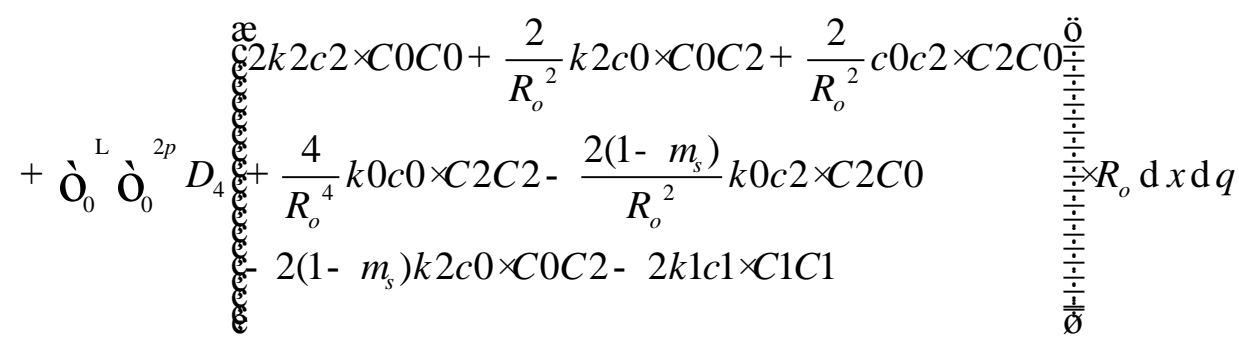

$$
\begin{aligned}
& +\left(k_{r 1}^{L} \times\left. k 0 c 0\right|_{x=0} \times C 0 C 0+K_{r 1}^{L} \times\left. k 1 c 1\right|_{x=0} \times C 0 C 0\right) \times R_{o} \\
& +\left(k_{r 1}^{R} \times\left. k 0 c 0\right|_{x=L} \times C 0 C 0+K_{r 1}^{R} \times\left. k 1 c 1\right|_{x=L} \times C 0 C 0\right) \times R_{o} \\
& +\left(k_{x 1}^{L} \times\left. k 0 c 0\right|_{x=0} \times C 0 C 0+k_{x 1}^{R} \times\left. k 1 c 1\right|_{x=L} \times C 0 C 0\right) \times R_{o}
\end{aligned}
$$




$$
\mathbf{K}_{s l w w}^{21}=\mathbf{K}_{s l w w}^{12}
$$

$$
\begin{aligned}
& \mathbf{K}_{s 1 w w}^{22}=\grave{\mathrm{O}}_{0}^{L} \grave{\mathrm{O}}_{0}{ }_{0}^{2 p} \frac{2 D_{3}}{R^{2}} k 0 k 0 \times C 0 C 0 \times R_{o} \mathrm{~d} x \mathrm{~d} q
\end{aligned}
$$

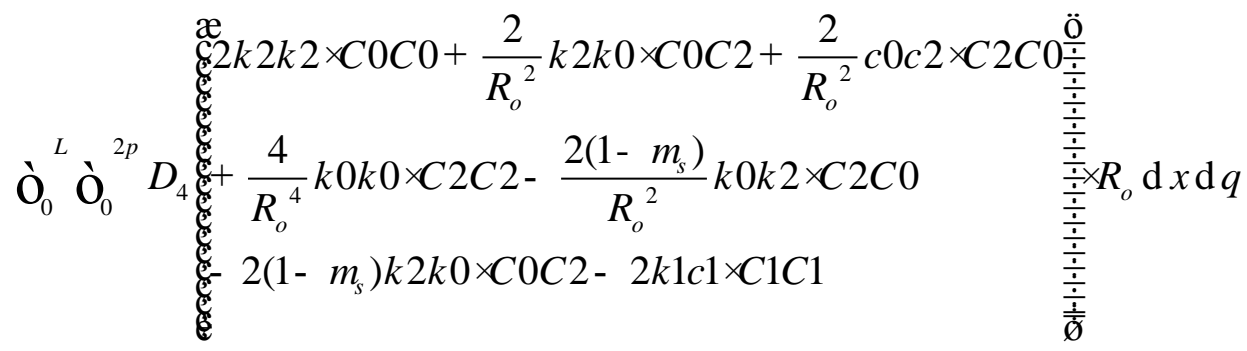

$$
\begin{aligned}
& +\left(k_{r 1}^{L} \times\left. k 0 k 0\right|_{x=0} \times C 0 C 0+K_{r 1}^{L} \times\left. k 1 c 1\right|_{x=0} \times C 0 C 0\right) \times R_{1} \\
& +\left(k_{r 1}^{R} \times\left. k 0 k 0\right|_{x=L} \times C 0 C 0+K_{r 1}^{R} \times\left. k 1 c 1\right|_{x=L} \times C 0 C 0\right) \times R_{1} \\
& +\left(k_{r 1}^{c} \times\left. k 0 k 0\right|_{x=0} \times C 0 C 0+K_{r 1}^{c} \times\left. k 1 c 1\right|_{x=0} \times C 0 C 0\right) \times R_{1} \\
& \mathbf{K}_{p s 1, u w}^{11}=-k_{x 1}^{c} \times R_{o} \times\left. c 0 c 0\right|_{s=b, x=x c} \grave{\mathrm{O}}_{0}^{2 p} C 0 C 0 \mathrm{~d} q \\
& \mathbf{K}_{p s 1, u w}^{12}=-k_{x 1}^{c} \times R_{o} \times\left. c 0 k 0\right|_{s=b, x=x c} \times \grave{\mathbf{O}}_{0}^{2 p} C 0 C 0 \mathrm{~d} q \\
& \mathbf{K}_{p s, 1, u w}^{21}=-k_{x 1}^{c} \times R_{o} \times\left. f 0 c 0\right|_{s=b, x=x c} \times \grave{\mathrm{O}}_{0}^{2 p} C 0 C 0 \mathrm{~d} q \\
& \mathbf{K}_{p s 1, u w}^{22}=-k_{x 1}^{c} \times R_{o} \times\left. f 0 k 0\right|_{s=b, x=x c} \times \grave{\mathbf{O}}_{0}^{2 p} C 0 C 0 \mathrm{~d} q
\end{aligned}
$$

$$
\begin{aligned}
& \mathbf{K}_{p s 1, v v}^{11}=-k_{q 1}^{c} \times R_{o} \times\left. c 0 c 0\right|_{s=b, x=x c} \times \grave{\mathbf{O}}_{0}^{2 p} S 0 S 0 \mathrm{~d} q \\
& \mathbf{K}_{p s 1, v v}^{12}=-k_{q 1}^{c} \times R_{o} \times\left. f 0 c 0\right|_{s=b, x=x c} \times \grave{\mathbf{O}}_{0}^{2 p} S 0 S 0 \mathrm{~d} q \\
& \mathbf{K}_{p s 1, v v}^{21}=-k_{q 1}^{c} \times R_{0} \times\left. c 0 f 0\right|_{s=b, x=x c} \times \grave{\mathbf{O}}_{0}^{2 p} S 0 S 0 \mathrm{~d} q \\
& \mathbf{K}_{p s 1, v v}^{22}=-k_{q 1}^{c} \times R_{o} \times\left. f 0 f 0\right|_{s=b, x=x c} \times \grave{\mathbf{O}}_{0}^{2 p} S 0 S 0 \mathrm{~d} q \\
& \mathbf{K}_{p s 1, w w}^{11}=-k_{x 1}^{c} \times R_{o} \times\left. c 1 c 1\right|_{s=b, x=x c} \times \grave{\mathbf{O}}_{0}^{2 p} C 0 C 0 \mathrm{~d} q \\
& \mathbf{K}_{p s 1, w w}^{12}=-k_{x 1}^{c} \times R_{o} \times\left. f 1 c 1\right|_{s=b, x=x c} \times \grave{\mathbf{O}}_{0}^{2 p} C 0 C 0 \mathrm{~d} q
\end{aligned}
$$




$$
\begin{aligned}
& \mathbf{K}_{p s 1, w w}^{21}=-k_{x 1}^{c} \times R_{o} \times\left. c 1 k 1\right|_{s=b, x=x c} \times \grave{\mathbf{O}}_{0}^{2 p} C 0 C 0 \mathrm{~d} q \\
& \mathbf{K}_{p s 1, w w}^{22}=-k_{x 1}^{c} \times R_{o} \times\left. f 1 k 1\right|_{s=b, x=x c} \times \grave{\mathrm{O}}_{0}^{2 p} C 0 C 0 \mathrm{~d} q \\
& \mathbf{M}_{p u u}^{11}=P_{1} \times \grave{\mathbf{O}}_{0}^{b} \grave{\mathbf{O}}_{0}^{2 p} c 0 c 0 \times C 0 C 0 \times(s+a) \mathrm{d} q \mathrm{~d} s \\
& \mathbf{M}_{p u u}^{12}=P_{1} \grave{\mathbf{O}}_{0}^{b} \grave{\mathbf{O}}_{0}^{2 p} c 0 f 0 \times C 0 C 0 \times(s+a) \mathrm{d} q \mathrm{~d} s \\
& \mathbf{M}_{p u u}^{21}=\mathbf{M}_{p u u}^{12} \\
& \mathbf{M}_{p u u}^{22}=P_{1} \times \grave{\mathbf{O}}_{0}^{b} \grave{\mathbf{O}}_{0}^{2 p} f 0 f 0 \rtimes C 0 C 0 \times(s+a) \mathrm{d} q \mathrm{~d} s \\
& \mathbf{M}_{p v v}^{11}=P_{1} \times \grave{\mathrm{O}}_{0}^{b} \grave{\mathrm{O}}_{0}^{2 p} c 0 c 0 \times S 0 S 0 \times(s+a) \mathrm{d} q \mathrm{~d} s \\
& \mathbf{M}_{p v v}^{12}=P_{1} \times \grave{\mathbf{O}}_{0}^{b} \grave{\mathbf{O}}_{0}^{2 p} c 0 f 0 \times \operatorname{SOS} 0 \times(s+a) \mathrm{d} q \mathrm{~d} s \\
& \mathbf{M}_{p w v}^{21}=\mathbf{M}_{p v v}^{12} \\
& \mathbf{M}_{p v v}^{22}=P_{1} \times \grave{\mathbf{O}}_{0}^{b} \grave{\mathbf{O}}_{0}^{2 p} f 0 f 0 \times \operatorname{SOS} 0 \times(s+a) \mathrm{d} q \mathrm{~d} s \\
& \mathbf{M}_{p w w}^{11}=P_{1} \times \grave{\mathbf{O}}_{0}^{b} \grave{\mathbf{O}}_{0}^{2 p} c 0 c 0 \times C 0 C 0 \rtimes(s+a) \mathrm{d} q \mathrm{~d} s \\
& \mathbf{M}_{p w w}^{12}=P_{1} \times \grave{\mathbf{O}}_{0}^{b} \grave{\mathrm{O}}_{0}^{2 p} c 0 f 0 \times C 0 C 0 \rtimes(s+a) \mathrm{d} q \mathrm{~d} s \\
& \mathbf{M}_{p w w}^{21}=\mathbf{M}_{p w w}^{12} \\
& \mathbf{M}_{p w w}^{22}=P_{1} \times \grave{\mathbf{O}}_{0}^{b} \grave{\mathbf{O}}_{0}^{2 p} f 0 f 0 \times C 0 C 0 \times(s+a) \mathrm{d} q \mathrm{~d} s
\end{aligned}
$$

where,

$$
\begin{aligned}
& D_{1}=\frac{E h_{p}}{2\left(1-m^{2}\right)}, D_{2}=\frac{E h_{p}{ }^{3}}{24\left(1-m^{2}\right)}, D_{3}=\frac{E h_{s}}{2\left(1-m^{2}\right)}, D_{4}=\frac{E h_{s}{ }^{3}}{24\left(1-m^{2}\right)} \\
& P_{1}=\frac{r h_{p}}{2}, P_{2}=\frac{r h_{s}}{2}
\end{aligned}
$$

The unknown symbols are defined in the following forms: 


$$
C 0 C 0=\cos (m q) \cos (n q)
$$

$$
S O S 0=\sin (m q) \sin (n q)
$$

$C 2 C 0=-m^{2} \cos (m q) \cos (n q)$

$$
C 0 C 2=-n^{2} \cos (m q) \cos (n q)
$$

$$
C 1 C 1=m n \sin (m q) \sin (n q)
$$

$$
C 2 S 1=-m^{2} n \cos (m q) \cos (n q)
$$

$$
C 1 S 0=-m \sin (m q) \sin (n q)
$$

$$
S 0 C 1=-n \sin (m q) \sin (n q)
$$

$$
c 1 c 1=l_{m} l_{n} \sin \left(l_{m} s\right) \sin \left(l_{n} s\right)
$$

$$
c 0 c 0=\cos \left(l_{m} s\right) \cos \left(l_{n} s\right)
$$

$c 1 c 0=-l_{m} \sin \left(l_{m} s\right) \cos \left(l_{n} s\right)$

$$
c 2 c 1=l_{m}^{2} l_{n} \cos \left(l_{m} s\right) \sin \left(l_{n} s\right)
$$

$$
c 1 c 2=l_{m} l_{n}^{2} \cos \left(l_{m} s\right) \sin \left(l_{n} s\right)
$$

$$
f 1 c 0=x^{(1)}(s) \times \cos \left(l_{m} s\right)
$$

$f 1 c 1=x^{(1)}(s) \succ\left(-l_{m} \sin \left(l_{m} s\right)\right)$

$$
f 1 f 1=x^{(1)}(s) \times x^{(1)}(s)
$$

$$
k 1 c 0=z^{(1)}(s) \times \cos \left(l_{m} s\right)
$$

$$
k 1 c 1=z^{(1)}(s) \rtimes\left(-l_{m} \sin \left(l_{m} s\right)\right)
$$


where, $l_{m}=l_{m p}=\frac{m p}{b}, l_{n}=l_{n p}=\frac{n p}{b}$ for the annular plate and $l_{m}=l_{m s}=\frac{m p}{L}, l_{n}=l_{n s}=\frac{n p}{L}$ for the cylindrical shell.

\section{References}

[1]Leissa AW. Vibration of shells. Reported LC-77-186367, NASA-sp-288; 1973.

[2]Junger MC, Feit D. Sound, structures, and their interaction. Cambridge: MIT press, 1986.

[3]Hua L, Lam KY. Frequency characteristics of a thin rotating cylindrical shell using the generalized differential quadrature method. Int J Mech Sci 1998; 40(5): 443-459.

[4]Zhang XM, Liu GR, Lam KY. Vibration analysis of thin cylindrical shells using wave propagation approach. J Sound Vib 2001; 239(3): 397-403.

[5]Amabili M, Paidoussis MP. Review of studies on geometrically nonlinear vibrations and dynamics of circular cylindrical shells and panels, with and without fluid-structure interaction. Appl Mech Rev 2003; 56(4): 349-381.

[6]K-Uuml TV, R-Uuml, Tsien HS. The Buckling of Thin Cylindrical Shells under Axial Compression. J Spacecraft Rockets 2012; 40(6):165-181.

[7]Chen Y, Jin G, Liu Z. Free vibration analysis of circular cylindrical shell with non-uniform elastic boundary constraints. Int J Mech Sci 2013; 74: 120-132.

[8]Jin G, Ye T, Chen Y, Su Z, Yan Y. An exact solution for the free vibration analysis of laminated composite cylindrical shells with general elastic boundary conditions. Compos Struct 2013; 106(12):114-127.

[9]Hoppmann WH. Some characteristics of the flexural vibrations of orthogonally stiffened cylindrical shells. J Acoust Soc Am1958; 30: 77-82. 
[10]Mikulas Jr MM, McElman JA. On the free vibration of eccentrically stiffened cylindrical shells and plates. NASA TN-D 3010;165.

[11]Chen M, Zhang C, Tao X, et al. Structural and acoustic responses of a submerged stiffened conical shell. Shock Vib, 2014.

[12]Jafari AA, Bagheri M. Free vibration of non-uniformly ring stiffened cylindrical shells using analytical, experimental and numerical methods. Thin Wall Struct 2006; 44(1): 82-90.

[13]Mustafa BAJ, Ali R. An energy method for free vibration analysis of stiffened circular cylindrical shells. Comput Struct 1989; 32(2): 355-363.

[14] Wei JX, Chen MX, Hou GX, Xie K, Deng NQ. Wave based method for free vibration analysis of cylindrical shells with nonuniform stiffener distribution. J Vib Acoust 2013; 135(6): 26272637.

[15]Biron A. Limit analysis of cylindrical shells with longitudinal rib reinforcements. Int J Solids Struct 1970; 6: 893-908.

[16] Maskeliūnas, Danielius. Method reduction of vibrations of a cylindrical shell with a longitudinal plate rib. Solid State Phenom 2006; 113: 265-270.

[17]Wang JTS, Rinehart SA. Free vibrations of longitudinally stiffened cylindrical shells. J Appl Mech 1975; 41(4): 1087-1093.

[18] Averbukh AZ, Mavlyutov IG. Vibrations of a cylindrical shell with longitudinal ribs not reaching its edges. Soviet Appl Mech 1988; 24(1): 32-37.

[19] Vanlaere W, Impe RV, Lagae G. Influence of dimensions of longitudinal stiffeners on the buckling behaviour of locally supported cylindrical. The Ninth East Asia-Pasific Conference on Structural Engineering and Construction Proceedings, 2003. 
[20] Koval LR. On sound transmission into a stiffened cylindrical shell with rings and stringers treated as discrete elements. J Sound Vib 1980; 71(4):511-521.

[21]Kim YW, Lee YS, Ko SH. Coupled vibration of partially fluid-filled cylindrical shells with ring stiffeners. J Sound Vib 2004; 276(3-5):869-897.

[22]Zheng H, Zhou QD, Ji G. Influence of Rib Strengthening on Structure Vibration and Sound Radiation of Cylindrical Shell. J Appl Biomater Biom 2012; 226-228:359-363.

[23]Tso YK, Hansen CH. Wave propagation through cylinder-plate junctions. J Sound Vib 1995; 186(3):447-461.

[24]Cheng L, Nicolas J. Free vibration analysis of a cylindrical shell-circular plate system with general coupling and various boundary conditions. J Sound Vib 1992; 155(2): 231-247.

[25]Cheng L. Fluid-structural coupling of a plate-ended cylindrical shell: vibration and Internal sound field. J Sound Vib 1994; 174(5):641-654.

[26]Chen M, Wei J, Xie K, Deng N, Hou G. Wave based method for free vibration analysis of ring stiffened cylindrical shell with intermediate large frame ribs. Shock Vib 2013; 20(3): 459-479.

[27]Qu Y, Chen Y, Long X, Hua H, Meng G. A modified variational approach for vibration analysis of ring-stiffened conical-cylindrical shell combinations. Eur J Mech A-Solid 2013; 37:200-215.

[28]Wu S, Qu Y, Hua H. Vibration characteristics of a spherical-cylindrical-spherical shell by a domain decomposition method. Mech Res Commun 2013; 49(3):17-26.

[29] Yan J, Zhang J. Power flow and sound radiation of a submerged cylindrical shell with internal structural. Appl Mech Mater 2011; 105-107: 321-325.

[30] Yamada G, Irie T, Tamiya T. Free vibration of a circular cylindrical double-shell system closed by end plates. J Sound Vib 1986; 108(2):297-304. 
[31]Li WL. Free vibration of beams with general boundary conditions. J Sound Vib 2000; 237(4):709-725.

[32]Jin GY, Chen H, Du JT, Yang TJ, Li WY. The influence of edge restraining stiffness on the transverse vibrations of rectangular plate structures. J Marine Sci Sppl; 2010; 9:494-402

[33] Shi SX, Jin GY, Liu ZG. Vibro-acoustic behaviors of an elastically restrained double-panel structure with an acoustic cavity of arbitrary boundary impedance. Appl Acoust 2014; 76(1):431444.

\section{List of Collected Table and Figure Captions}

Table 1 The corresponding spring stiffnesses for three types of classical end boundary conditions

Table 2 Convergence of frequencies for the double-shell structure coupled with one annular plate $(L$ $\left.=3 \mathrm{~m}, R_{o}=0.5 \mathrm{~m}, R_{i}=0.3 \mathrm{~m}, h_{s}=h_{p}=0.005 \mathrm{~m}, x_{1}=\mathrm{L} / 2\right)$.

Table 3 Natural frequencies of the clamped-free double-shell structure coupled with 2 annular plates $\left(L=1.2 \mathrm{~m}, R_{o}=0.4 \mathrm{~m}, R_{i}=0.3 \mathrm{~m}, h_{s}=h_{p}=0.005 \mathrm{~m}, x_{1}=0, x_{2}=0.6 \mathrm{~m}\right)$.

Table 4 Natural frequencies of the double-shell structure coupled with 3 annular plates $\left(L=1.2 \mathrm{~m}, R_{o}\right.$ $\left.=0.5 \mathrm{~m}, R_{i}=0.4 \mathrm{~m}, h_{s}=h_{p}=0.005 \mathrm{~m}, x_{1}=0.1 \mathrm{~m}, x_{2}=0.6 \mathrm{~m}, x_{3}=1.1 \mathrm{~m},\right)$.

Table 5 The lowest five natural frequencies of the double-shell structure with elastic coupling boundary conditions ( $L=3 \mathrm{~m}, R_{o}=0.5 \mathrm{~m}, R_{i}=0.3 \mathrm{~m}, h_{s}=h_{p}=0.005 \mathrm{~m}, x_{1}=\mathrm{L} / 2$ ). 
Table 6 The first seven natural frequencies of the double-shell structure with elastically supported end boundaries for different stiffness ( $\left.L=3 \mathrm{~m}, R_{o}=0.5 \mathrm{~m}, R_{i}=0.3 \mathrm{~m}, h_{s}=h_{p}=0.005 \mathrm{~m}, x_{1}=\mathrm{L} / 2\right)$.

Fig. 1 Geometry and co-ordinate systems of a double-shell structure

Fig. 2 Displacement, force and moment resultants of the circular cylindrical double-shell structure

Fig. 3 Several mode shapes for the outer shell of the double-shell structure under different end boundary conditions, (a): F-F, (b): S-S, (c): C-C.

Fig. 4 Variation of frequencies with different positions of the annular plate under certain end boundary conditions, (a): F-F, (b): S-S, (c): C-C.

Fig.5 (a): The 3rd mode shape under free supported boundary condition; (b): The 1th mode shape under simply supported boundary condition

Fig. 6 Effects on the natural frequency of the stiffnesses of the elastic boundaries for some lower order modes

Fig. 7 Effects on the natural frequency of the stiffnesses of the elastic boundaries for some higher order modes

Fig. 8 Exciting point displacement responses on the inner shell of the double-shell structure

Fig. 9 Transfer point displacement responses on the outer shell of the double-shell structure with $x_{e 1}$ $=0.6 \mathrm{~m}$

Fig. 10 Transfer point displacement responses on the inner shell of the double-shell structure with $x_{e 2}=0.35 \mathrm{~m}$ 
Fig. 11 Transfer point displacement responses on the outer shell of the double-shell structure with $x_{e 2}=0.35 \mathrm{~m}$

Fig. 12 Dynamic responses of radial displacement under external excitation with different thicknesses of annular plate

Fig. 13 Dynamic responses of circumferential displacement under external excitation with different thicknesses of annular plate

Fig. 14 Dynamic responses of axial displacement under external excitation with different thicknesses of annular plate

Fig. 15 Dynamic responses of radial displacement under external s with different width of annular plate

Fig. 16 Dynamic responses of circumferential displacement under external excitation with different width of annular plate

Fig. 17 Dynamic responses of axial displacement under external excitation with different width of annular plate 
Figures:
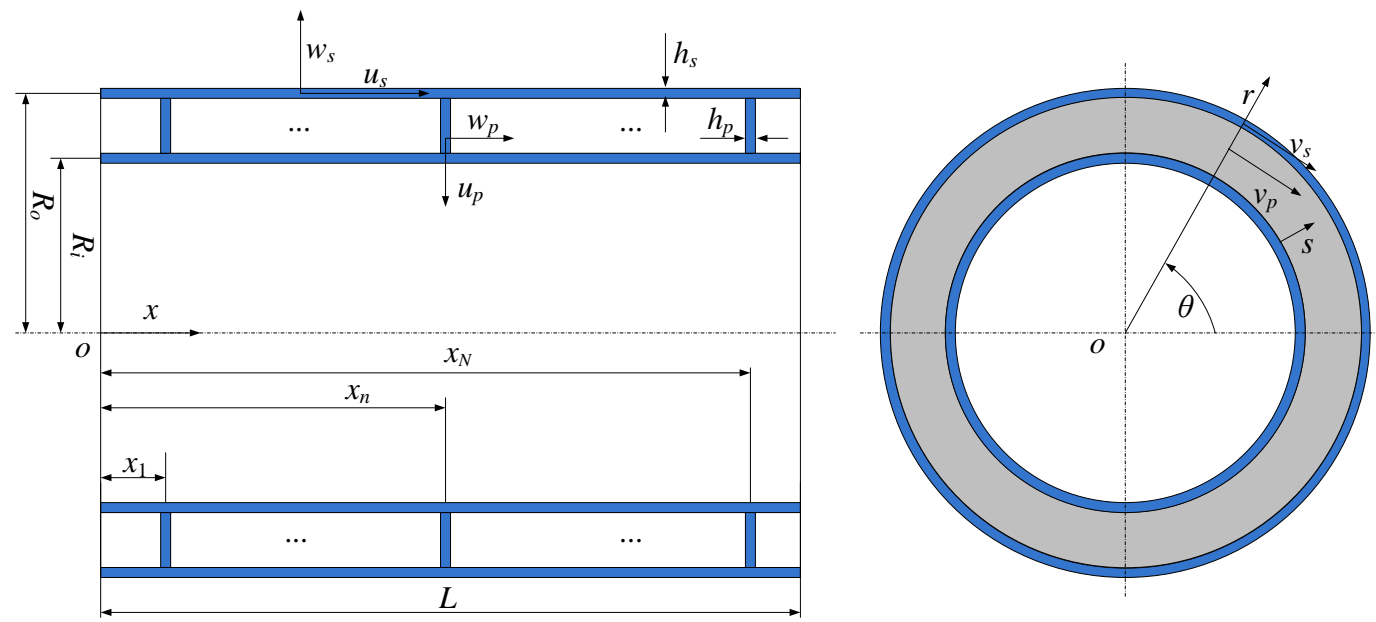

Fig. 1 Geometry and co-ordinate systems of a double-shell structure

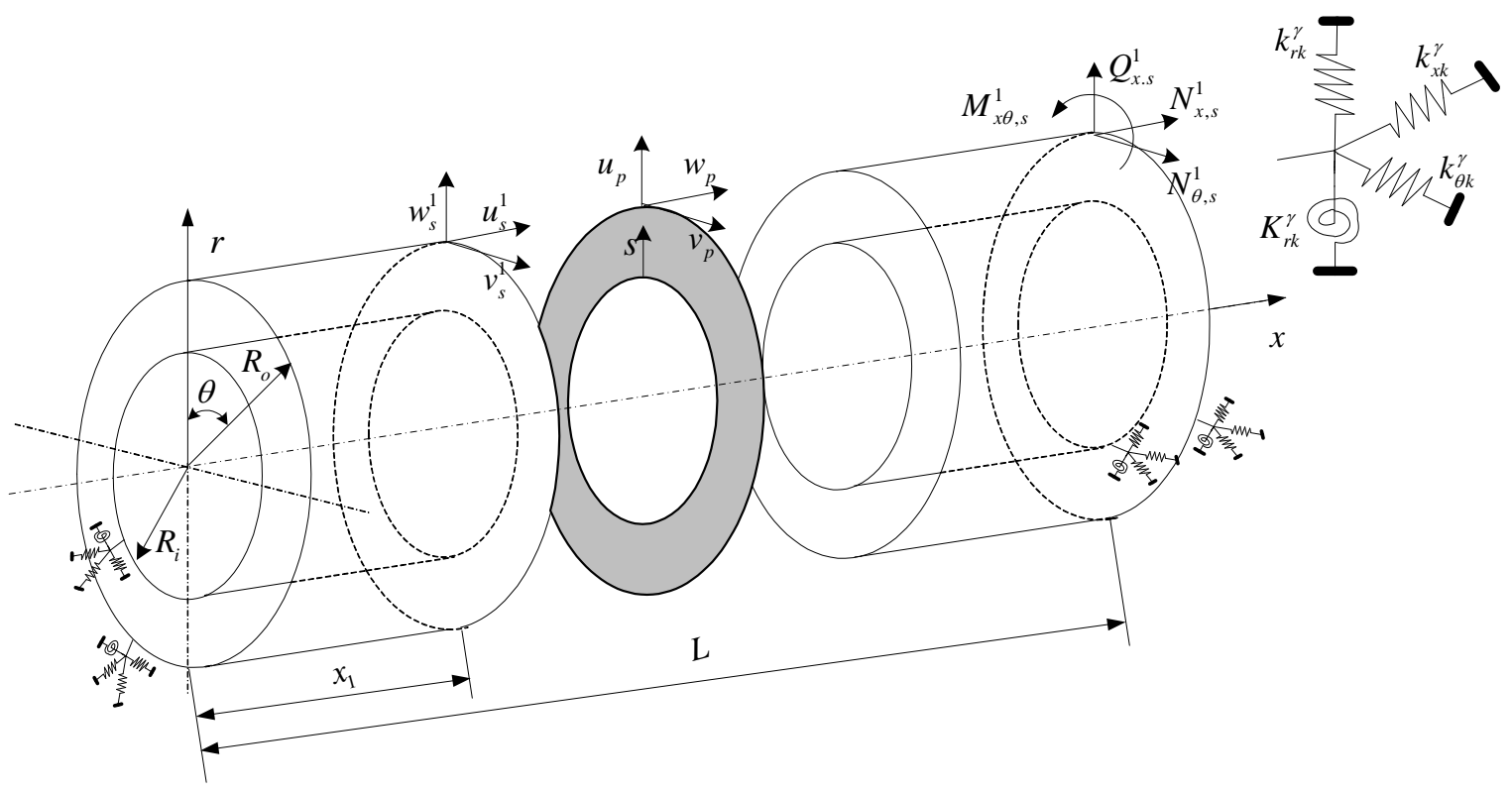

Fig. 2 Displacement, force and moment resultants of the circular cylindrical double-shell structure 


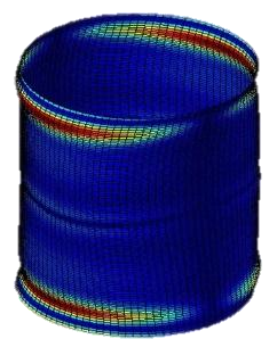

(a): $(1,1)$

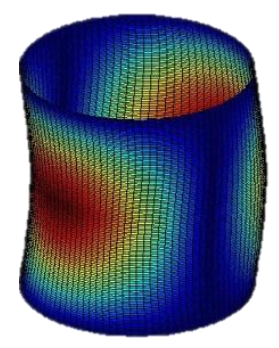

(b): $(1,1)$

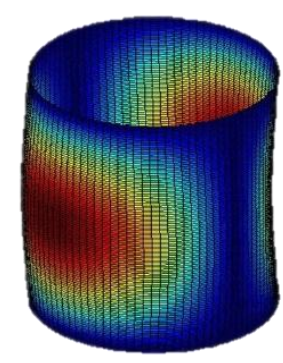

(c): $(1,1)$

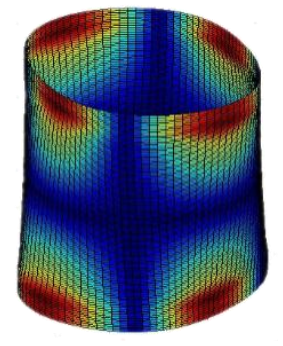

$(2,2)$

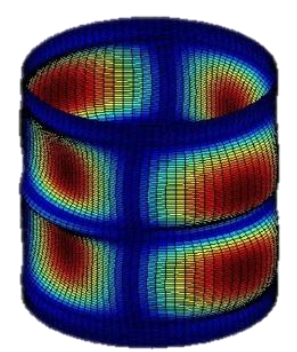

$(2,2)$

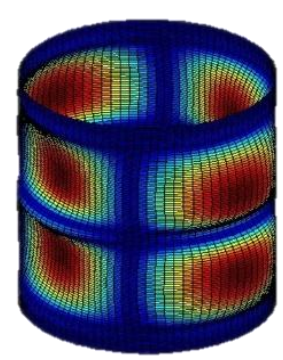

$(2,2)$

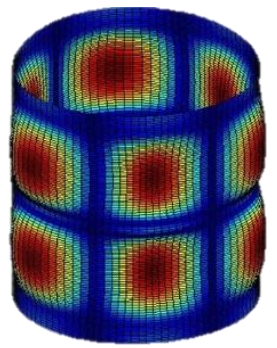

$(3,3)$

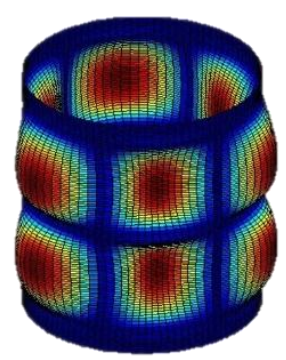

$(3,3)$

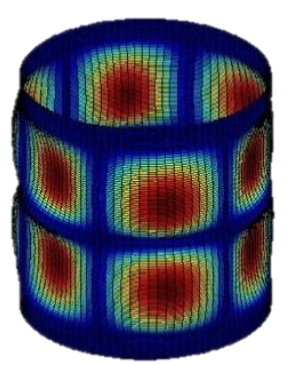

$(3,3)$

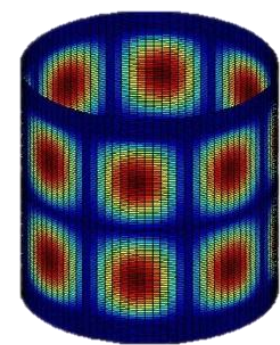

$(4,4)$

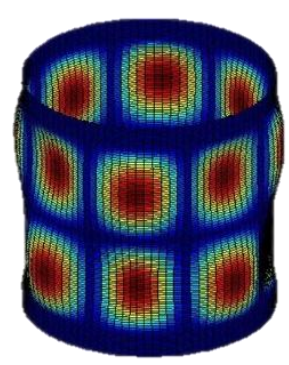

$(4,4)$

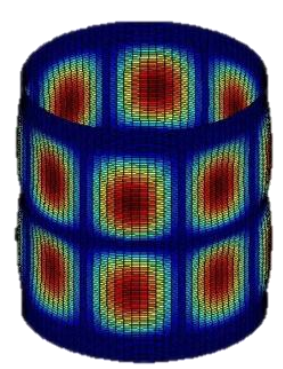

$(4,4)$

Fig. 3 Several mode shapes for the outer shell of the double-shell structure under different end boundary conditions, (a): F-F, (b): S-S, (c): C-C. 


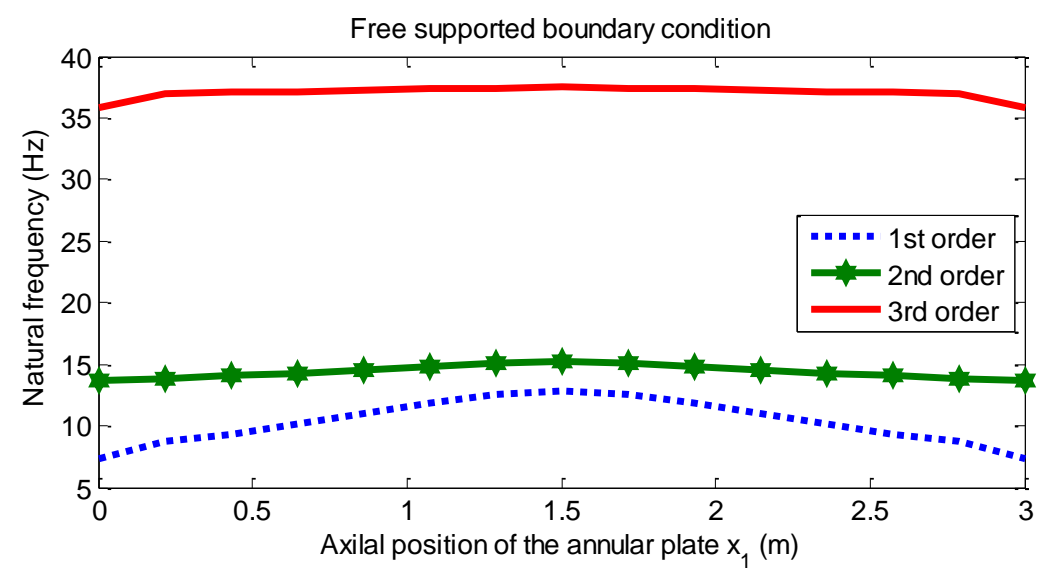

(a):

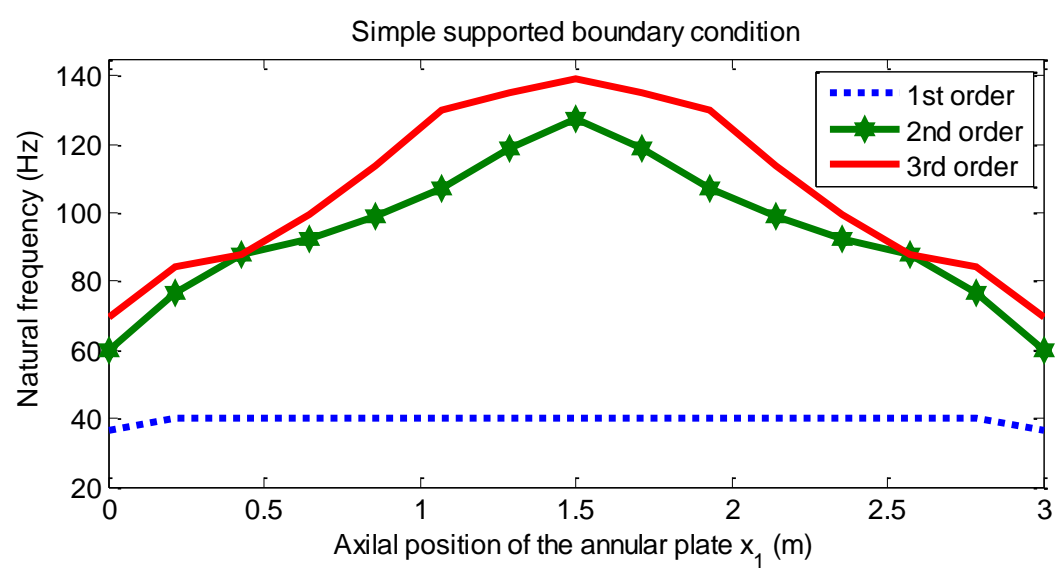

(b):

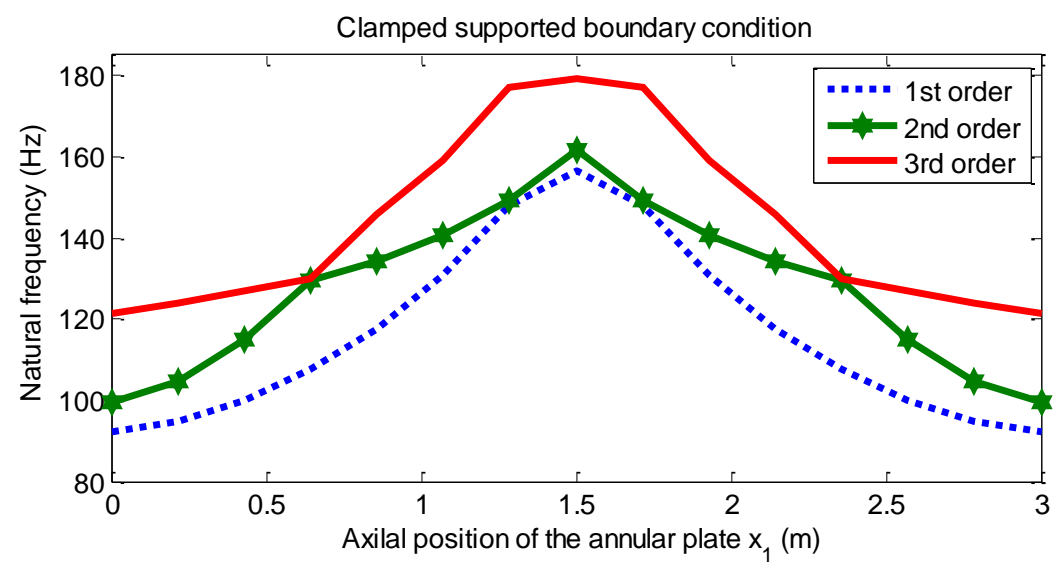

(c):

Fig. 4 Variation of frequencies with different positions of the annular plate under certain end boundary conditions, (a): F-F, (b): S-S, (c): C-C. 


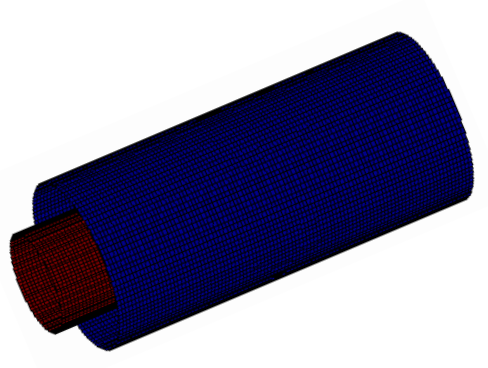

(a)

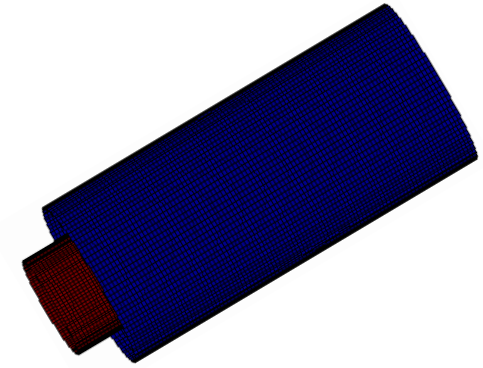

(b)

Fig. 5 (a): The 3rd mode shape under free supported boundary condition; (b): The 1th mode shape under simply supported boundary condition

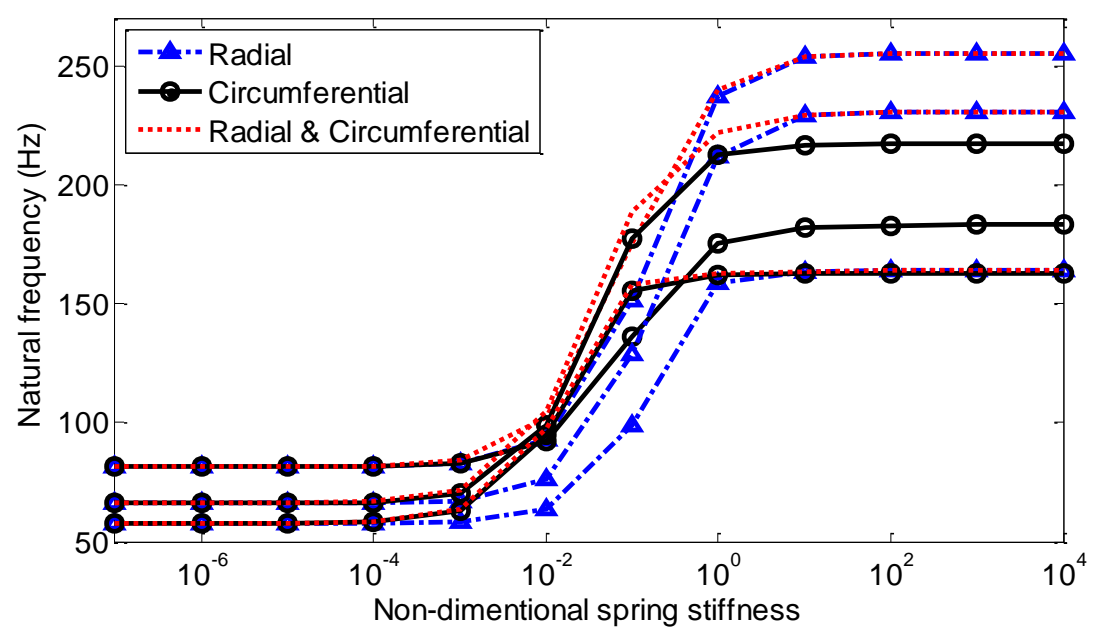


Fig. 6 Effects on the natural frequency of the stiffnesses of the elastic boundaries for some lower order modes

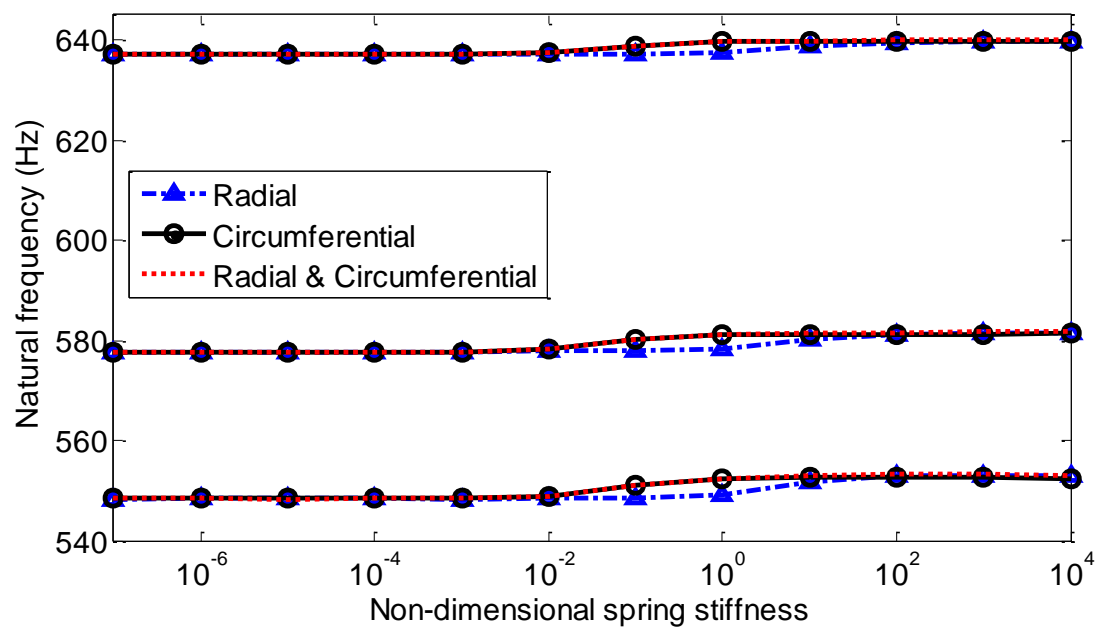

Fig. 7 Effects on the natural frequency of the stiffnesses of the elastic boundaries for some higher order modes

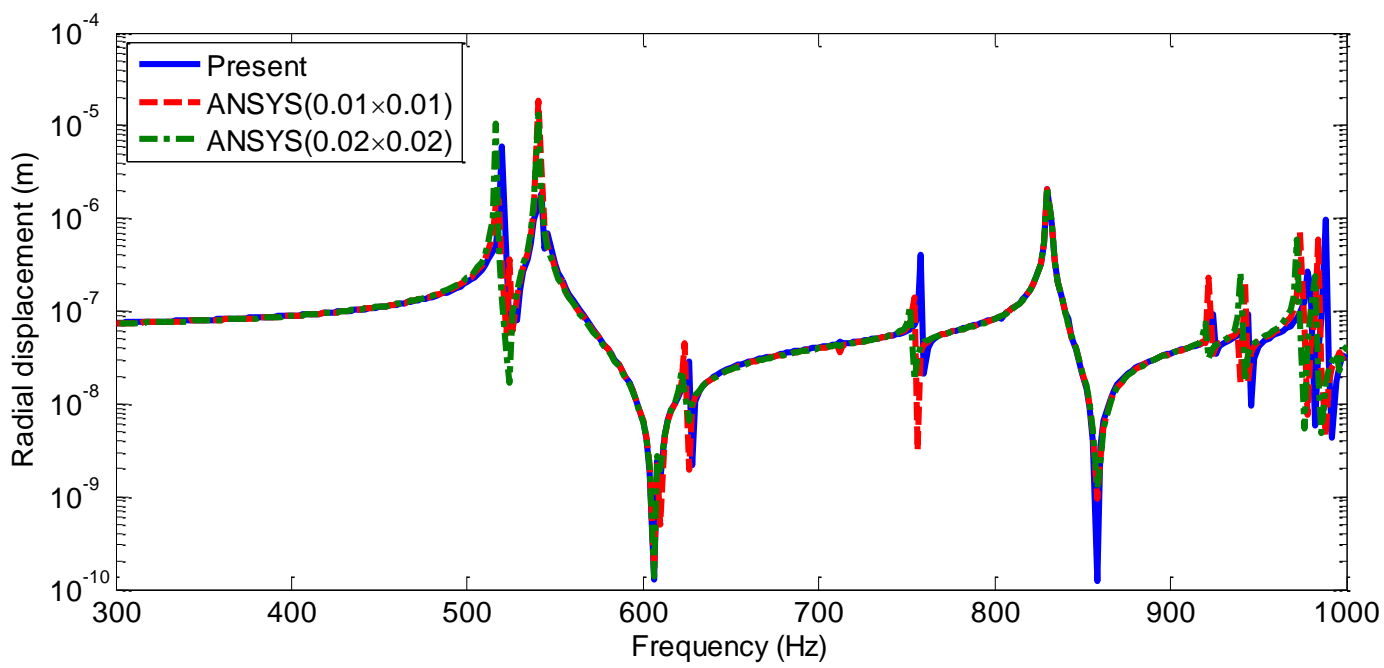

Fig. 8 Exciting point displacement responses on the inner shell of the double-shell structure 


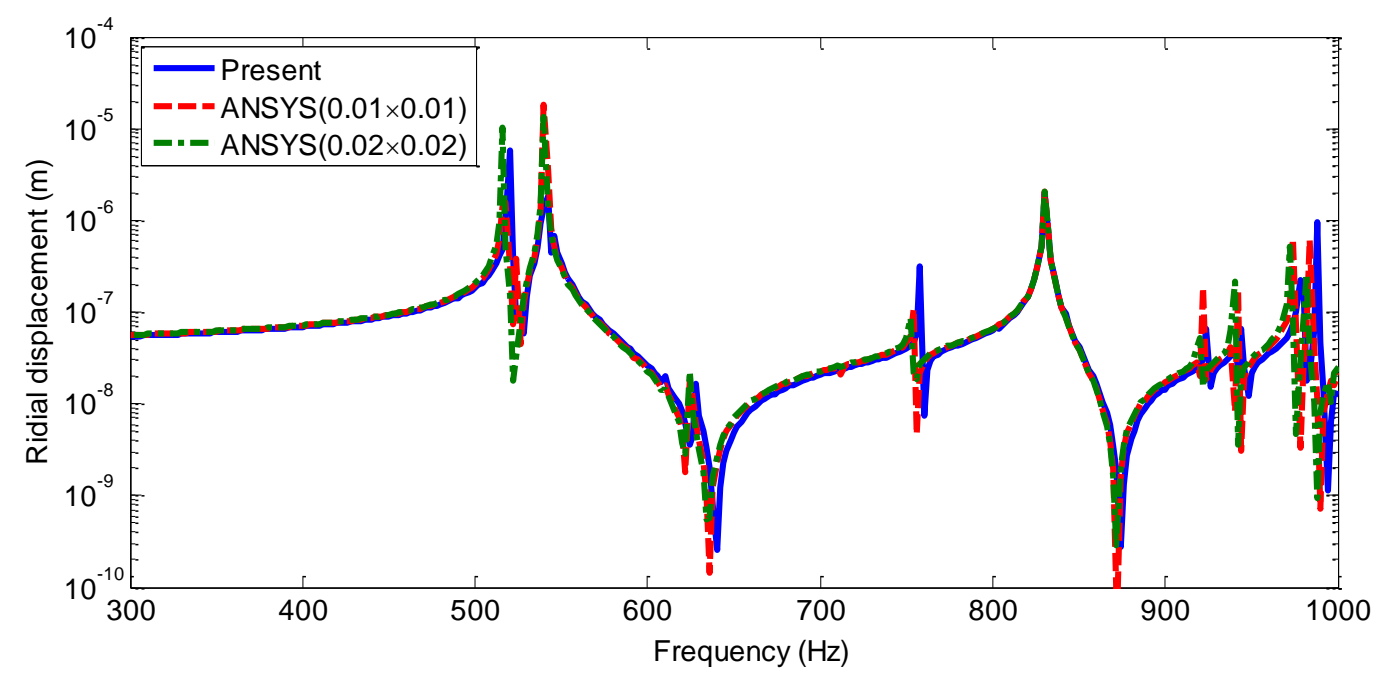

Fig. 9 Transfer point displacement responses on the outer shell of the double-shell structure with $x_{e 1}$ $=0.6 \mathrm{~m}$

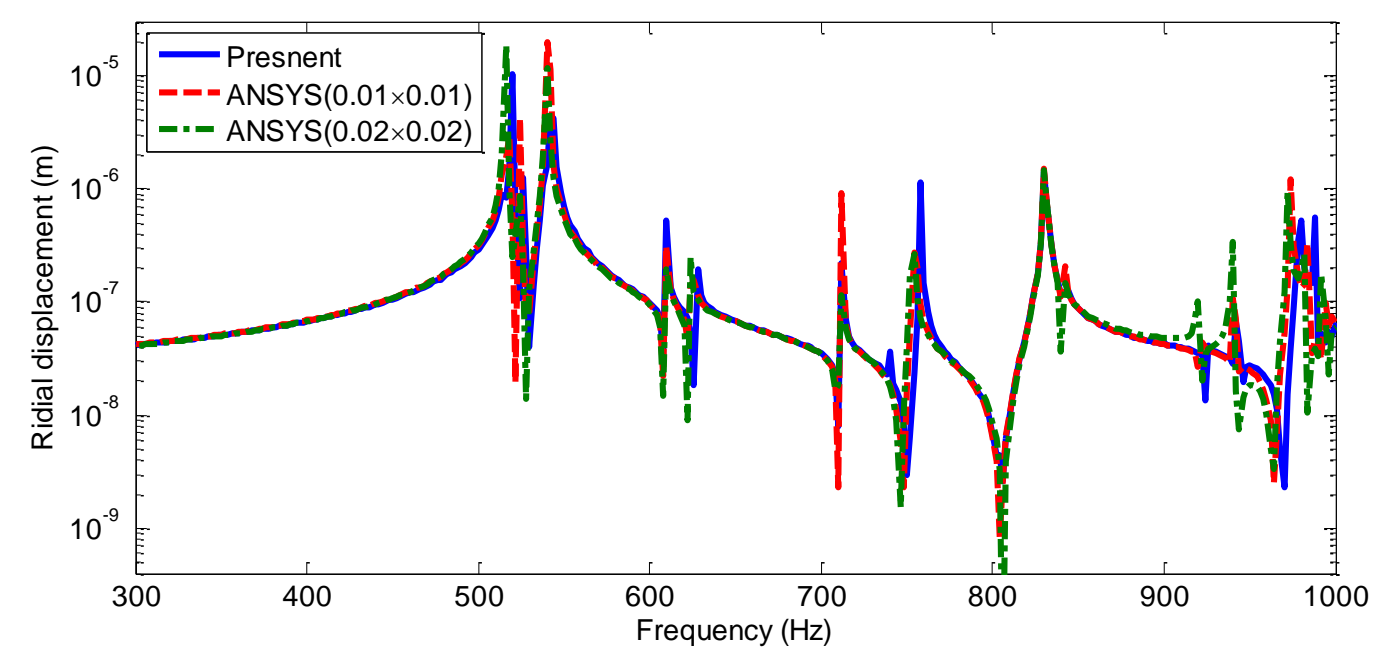

Fig. 10 Transfer point displacement responses on the inner shell of the double-shell structure with $x_{e 2}=0.35 \mathrm{~m}$ 


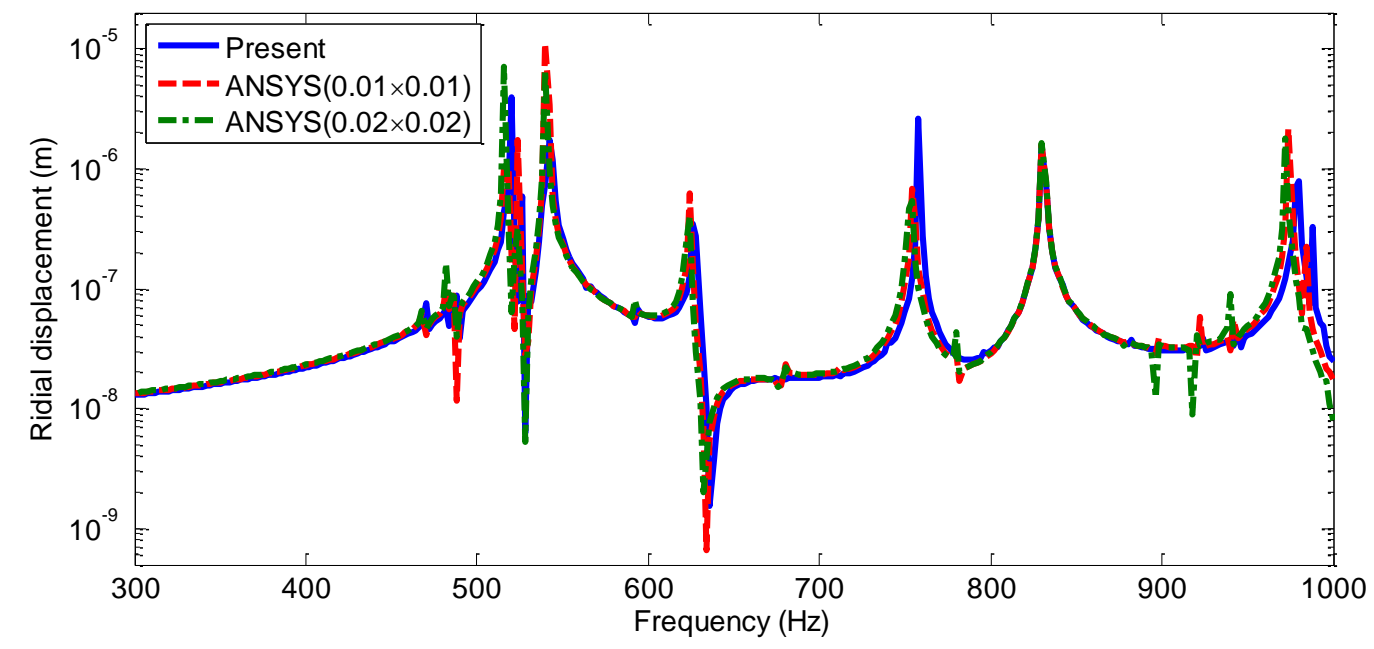

Fig. 11 Transfer point displacement responses on the outer shell of the double-shell structure with $x_{e 2}=0.35 \mathrm{~m}$

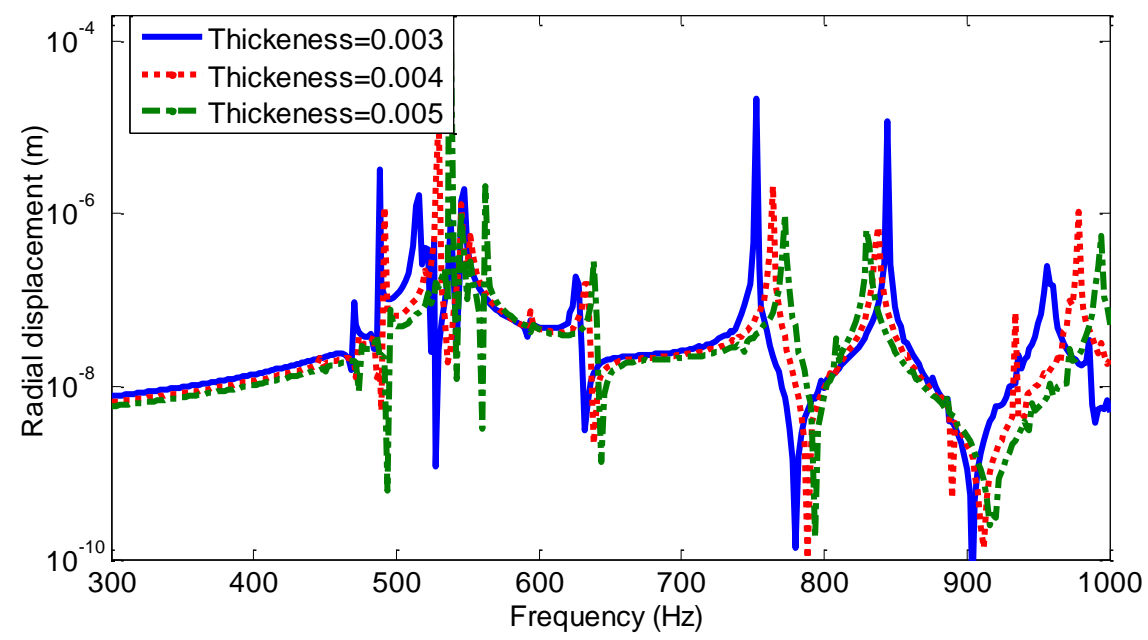

Fig. 12 Dynamic responses of radial displacement under external excitation with different thicknesses of annular plate 


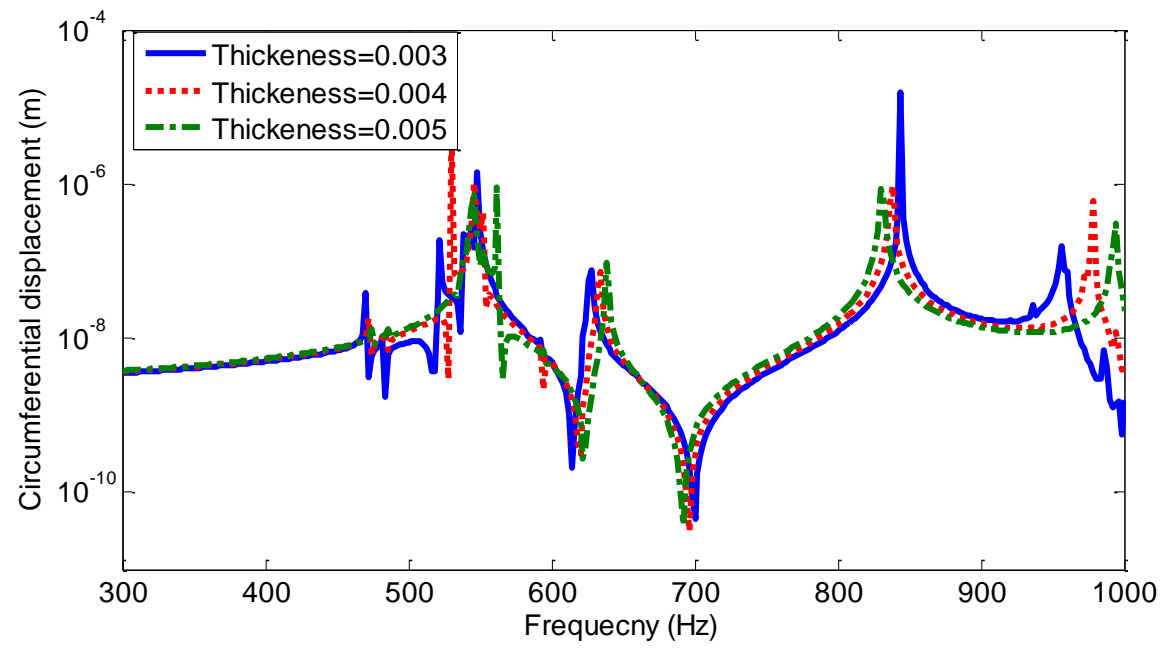

Fig. 13 Dynamic responses of circumferential displacement under external excitation with different thicknesses of annular plate

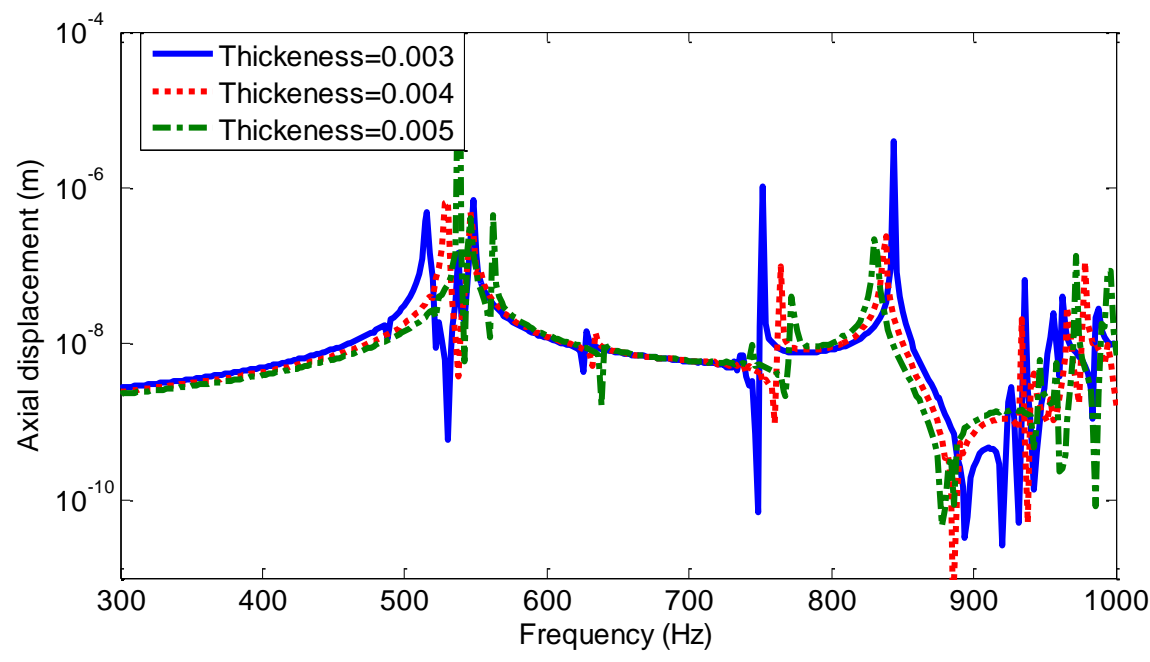

Fig. 14 Dynamic responses of axial displacement under external excitation with different thicknesses of annular plate 


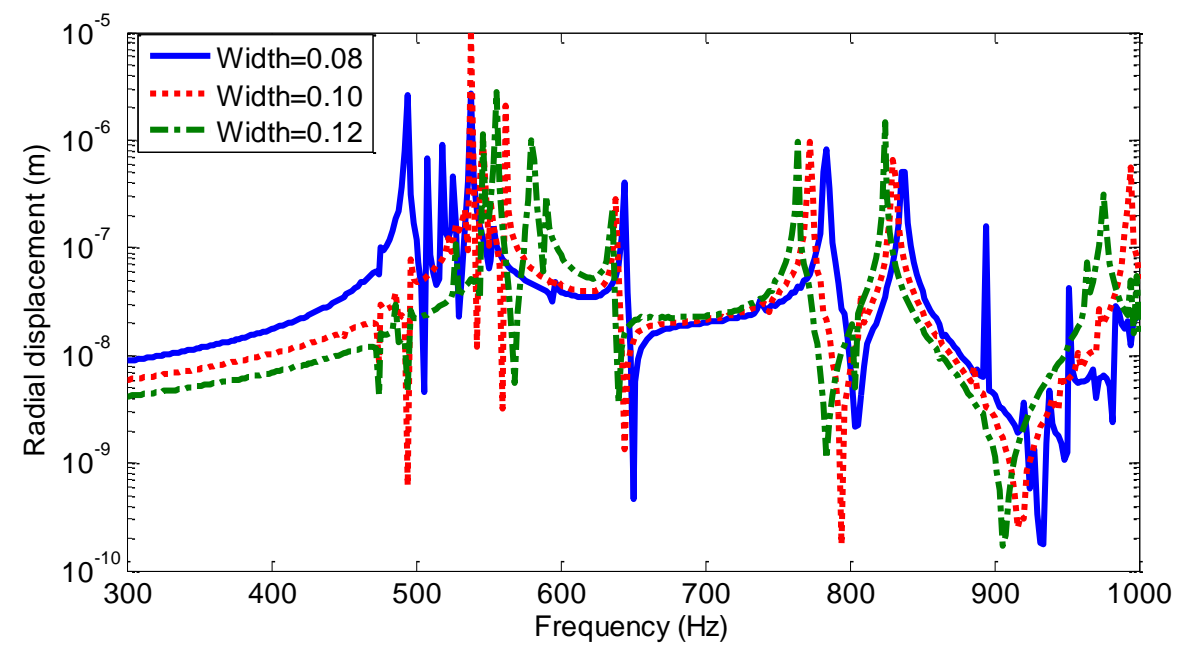

Fig. 15 Dynamic responses of radial displacement under external excitation with different width of annular plate

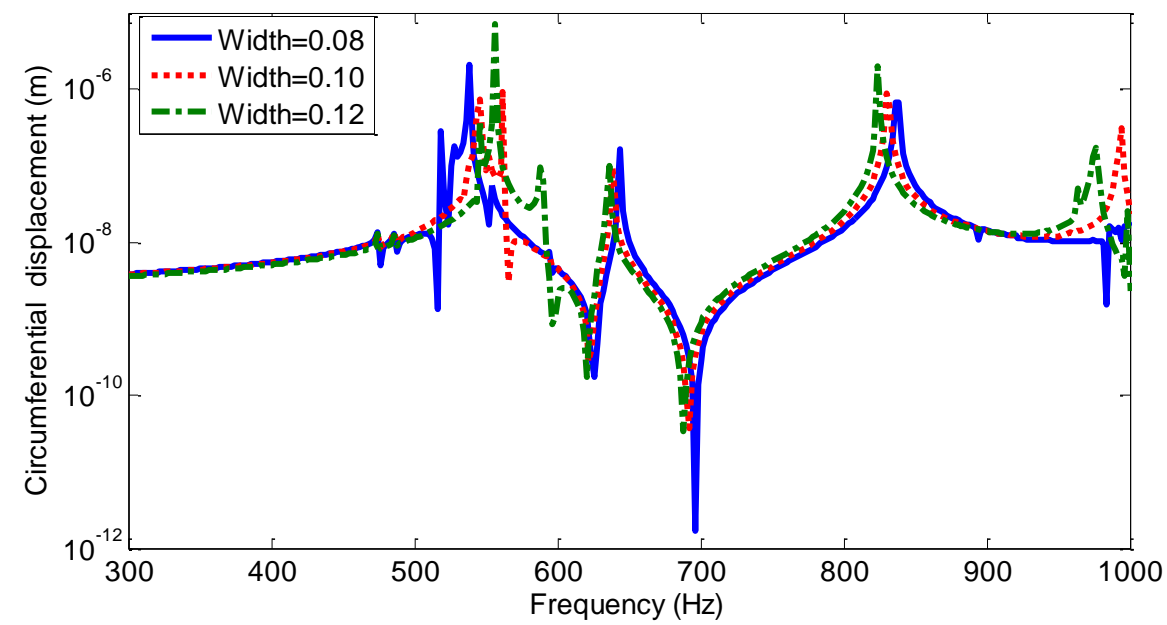

Fig. 16 Dynamic responses of circumferential displacement under external excitation with different width of annular plate 


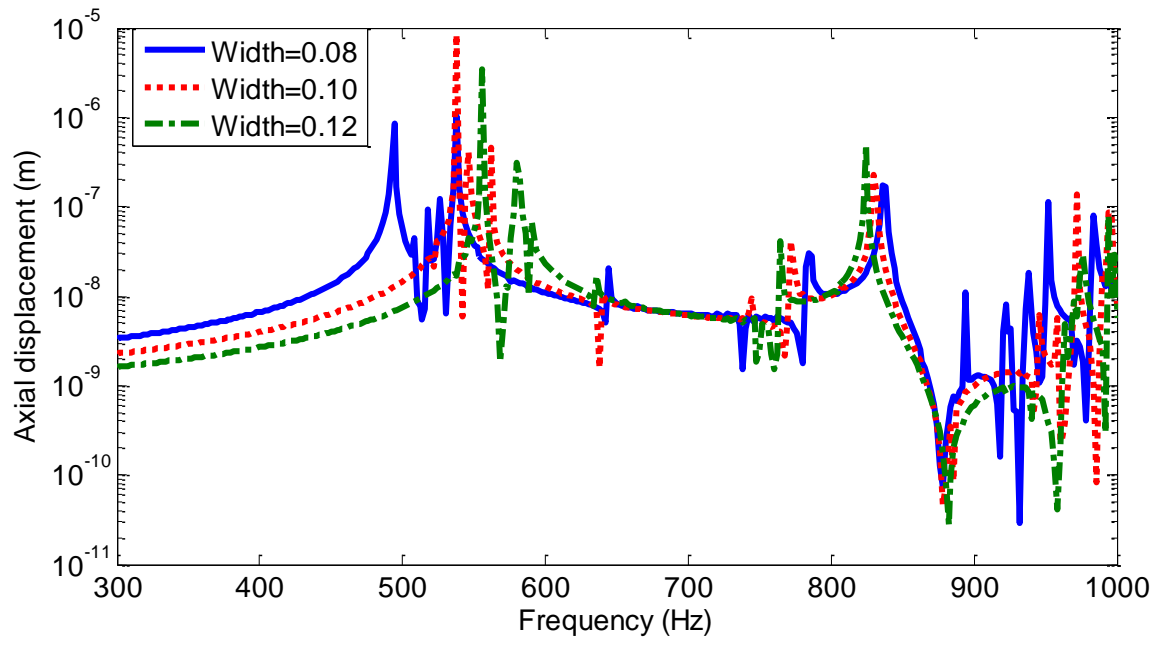

Fig. 17 Dynamic responses of axial displacement under external excitation with different width of annular plate 
Tables:

Table 1 The corresponding spring stiffnesses for three types of classical end boundary conditions

\begin{tabular}{cccc}
\hline Boundary & \multicolumn{3}{c}{ End boundary Conditions } \\
\cline { 2 - 4 } springs & $\mathrm{F}$ & $\mathrm{S}$ & $\mathrm{C}$ \\
\hline$k_{x}$ & 0 & 0 & $10^{13}$ \\
$k_{\theta}$ & 0 & $10^{13}$ & $10^{13}$ \\
$k_{r}$ & 0 & $10^{13}$ & $10^{13}$ \\
$K_{r}$ & 0 & 0 & $10^{13}$ \\
\hline
\end{tabular}




\section{Table 2}

Convergence of frequencies for the double-shell structure coupled with one annular plate $(L=3 \mathrm{~m}$, $\left.R_{o}=0.5 \mathrm{~m}, R_{i}=0.3 \mathrm{~m}, h_{s}=h_{p}=0.005 \mathrm{~m}, x_{1}=\mathrm{L} / 2\right)$.

\begin{tabular}{lccccccc}
\hline \multirow{2}{*}{ E.B. } & $n$ & \multicolumn{7}{c}{$m=1$} \\
\cline { 2 - 7 } & 1 & 304.80 & 304.80 & 304.32 & 304.30 & 304.18 & 304.18 \\
& 2 & 295.60 & 294.60 & 294.55 & 294.00 & 293.90 & 293.50 \\
C-C & 3 & 192.00 & 191.60 & 191.50 & 191.20 & 191.20 & 191.00 \\
& 4 & 162.50 & 162.10 & 162.01 & 161.80 & 161.74 & 161.54 \\
& 5 & 157.00 & 156.80 & 156.72 & 156.60 & 156.57 & 156.47 \\
& 6 & 190.50 & 190.40 & 190.38 & 190.30 & 190.30 & 190.25 \\
& 7 & 246.70 & 246.70 & 246.63 & 246.60 & 246.58 & 246.55 \\
& & & & & & & \\
F-F & 1 & 12.11 & 12.11 & 12.03 & 12.00 & 12.00 & 12.01 \\
& 2 & 15.23 & 15.22 & 15.22 & 15.22 & 15.22 & 15.21 \\
& 3 & 38.63 & 38.61 & 38.61 & 38.59 & 38.59 & 38.57 \\
& 4 & 72.87 & 72.83 & 72.83 & 72.81 & 72.80 & 72.78 \\
& 5 & 117.10 & 117.10 & 117.07 & 117.00 & 117.03 & 116.98 \\
& 6 & 171.30 & 171.20 & 171.21 & 171.10 & 171.14 & 171.08 \\
& 7 & 235.30 & 235.20 & 235.21 & 235.10 & 235.13 & 235.13 \\
& & & & & & & \\
& 1 & 208.80 & 208.80 & 208.75 & 208.80 & 208.69 & 208.68 \\
& 2 & 220.00 & 220.00 & 219.99 & 220.00 & 219.99 & 219.99 \\
& 3 & 153.20 & 153.20 & 153.20 & 153.20 & 153.20 & 153.20 \\
& 4 & 127.50 & 127.50 & 127.46 & 127.50 & 127.46 & 127.45 \\
& 5 & 139.00 & 139.00 & 138.95 & 138.90 & 138.94 & 138.94 \\
& 6 & 182.20 & 181.20 & 181.16 & 181.20 & 181.15 & 181.15 \\
& 7 & 242.50 & 242.50 & 242.54 & 242.50 & 242.53 & 242.53 \\
\hline
\end{tabular}




\section{Table 3}

Natural frequencies of the clamped-free double-shell structure coupled with 2 annular plates $(L=1.2$ $\left.\mathrm{m}, R_{o}=0.5 \mathrm{~m}, R_{i}=0.4 \mathrm{~m}, h_{s}=h_{p}=0.005 \mathrm{~m}, x_{1}=0, x_{2}=0.6 \mathrm{~m}\right)$.

\begin{tabular}{|c|c|c|c|c|c|c|c|c|c|c|c|c|c|}
\hline \multirow[b]{2}{*}{ E.B } & \multirow[b]{2}{*}{$m$} & \multicolumn{3}{|c|}{$n=1$} & \multicolumn{3}{|c|}{$n=2$} & \multicolumn{3}{|c|}{$n=3$} & \multicolumn{3}{|c|}{$n=4$} \\
\hline & & Present & FEM & $\begin{array}{c}\text { Error } \\
\%\end{array}$ & Present & FEM & $\begin{array}{c}\text { Error } \\
\%\end{array}$ & Present & FEM & $\begin{array}{c}\text { Error } \\
\%\end{array}$ & Present & FEM & $\begin{array}{c}\text { Error } \\
\%\end{array}$ \\
\hline \multirow{8}{*}{$\mathrm{C}-\mathrm{F}$} & 1 & 342.4 & 342.4 & 0.01 & 234.1 & 233.9 & 0.06 & 382.5 & 381.3 & 0.32 & 411.2 & 413.7 & 0.62 \\
\hline & 2 & 954.8 & 954.0 & 0.08 & 601.2 & 603.4 & 0.36 & 557.5 & 557.9 & 0.08 & 474.7 & 471.1 & 0.77 \\
\hline & 3 & 1261.3 & 1256.9 & 0.35 & 840.0 & 835.0 & 0.61 & 618.7 & 614.4 & 0.70 & 514.1 & 513.5 & 0.12 \\
\hline & 4 & 1338.8 & 1344.0 & 0.39 & 953.4 & 953.8 & 0.04 & 698.5 & 697.3 & 0.17 & 549.7 & 548.5 & 0.21 \\
\hline & 5 & 1378.5 & 1381.1 & 0.19 & 1113.3 & 1117.6 & 0.38 & 849.5 & 854.4 & 0.57 & 827.7 & 828.9 & 0.14 \\
\hline & 6 & 1502.3 & 1521.2 & 1.24 & 1333.7 & 1336.7 & 0.22 & 1113.3 & 1113.8 & 0.05 & 953.6 & 953.2 & 0.04 \\
\hline & 7 & 1545.1 & 1542.6 & 0.16 & 1396.5 & 1391.9 & 0.33 & 1188.1 & 1187.4 & 0.06 & 1033.5 & 1033.3 & 0.02 \\
\hline & 8 & 1574.0 & 1573.7 & 0.01 & 1455.8 & 1458.1 & 0.16 & 1225.8 & 1227.7 & 0.16 & 1042.3 & 1042.8 & 0.05 \\
\hline
\end{tabular}




\section{Table 4}

Natural frequencies of the double-shell structure coupled with 3 annular plates $\left(L=1.2 \mathrm{~m}, R_{o}=0.5 \mathrm{~m}\right.$, $\left.R_{i}=0.4 \mathrm{~m}, h_{s}=h_{p}=0.005 \mathrm{~m}, x_{1}=0.1 \mathrm{~m}, x_{2}=0.6 \mathrm{~m}, x_{3}=1.1 \mathrm{~m},\right)$.

\begin{tabular}{|c|c|c|c|c|c|c|c|c|c|c|c|c|c|}
\hline \multirow[b]{2}{*}{ E.B } & \multirow[b]{2}{*}{$m$} & \multicolumn{3}{|c|}{$n=1$} & \multicolumn{3}{|c|}{$n=2$} & \multicolumn{3}{|c|}{$n=3$} & \multicolumn{3}{|c|}{$n=4$} \\
\hline & & Present & FEM & $\begin{array}{c}\text { Error } \\
\%\end{array}$ & Present & FEM & $\begin{array}{c}\text { Error } \\
\%\end{array}$ & Present & FEM & $\begin{array}{c}\text { Error } \\
\%\end{array}$ & Present & FEM & $\begin{array}{c}\text { Error } \\
\%\end{array}$ \\
\hline \multirow{8}{*}{ C-C } & 1 & 0.67 & 830.84 & 0.02 & 540.78 & 540.57 & 0.04 & 523.93 & 517.91 & 1.16 & 545.06 & 543.67 & 0.26 \\
\hline & 2 & 1382.7 & 1377.3 & 0.39 & 979.84 & 975.19 & 0.48 & 740.85 & 42.00 & 0.16 & 8.35 & 587.24 & 0.19 \\
\hline & 3 & 1385.5 & 1382.3 & 0.23 & 995.01 & 996.17 & 0.12 & 761.54 & 756.21 & 0.70 & 26.64 & 626.09 & 0.09 \\
\hline & 4 & 1536.2 & 1534.1 & 0.14 & 1083.9 & 1083.3 & 0.06 & 805.56 & 804.71 & 0.10 & 627.56 & 626.57 & 0.16 \\
\hline & 5 & 1570.1 & 1567.1 & 0.19 & 1324.1 & 1323.9 & 0.02 & 1138.9 & 1135.0 & 0.38 & 1048.5 & 1047.8 & 0.07 \\
\hline & 6 & 1602.1 & 1597.8 & 0.27 & 1472.9 & 1471.1 & 0.12 & 1290.8 & 1287.0 & 0.26 & 1130.7 & 1131.5 & 0.07 \\
\hline & 7 & 1676.8 & 1663.6 & 0.79 & 1481.9 & 1479.9 & 0.14 & 1318.5 & 1319.0 & 0.01 & 1176.8 & 1170.9 & 0.50 \\
\hline & 8 & 1.1 & 1684.4 & 0.40 & 1596.7 & 1593.9 & 0.18 & 1395.9 & 1397.1 & 0.09 & 1209.7 & 1214.5 & 0.40 \\
\hline \multirow{8}{*}{ S-S } & 1 & 773.99 & 774.31 & 0.04 & 428.31 & 427.85 & 0.11 & 445.11 & 443.01 & 0.48 & 502.42 & 501.33 & 0.22 \\
\hline & 2 & 974.97 & 973.03 & 0.20 & 950.53 & 944.75 & 0.61 & 687.93 & 689.70 & 0.26 & 541.87 & 545.29 & 0.63 \\
\hline & 3 & 1218.2 & 1215.6 & 0.21 & 972.67 & 974.36 & 0.17 & 722.33 & 718.60 & 0.53 & 596.94 & 594.84 & 0.35 \\
\hline & 4 & 1380.1 & 1372.6 & 0.55 & 1076.4 & 1075.4 & 0.09 & 785.97 & 785.31 & 0.13 & 601.94 & 600.24 & 0.28 \\
\hline & 5 & 1381.9 & 1382.1 & 0.01 & 1314.9 & 1315.4 & 0.04 & 1117.9 & 1117.1 & 0.06 & 1032.9 & 1031.9 & 0.10 \\
\hline & 6 & 1531.3 & 1529.3 & 0.13 & 1472.2 & 1470.2 & 0.14 & 1286.7 & 1286.0 & 0.08 & 1127.6 & 1128.1 & 0.04 \\
\hline & 7 & 1567.9 & 1565.4 & 0.16 & 1479.7 & 1476.6 & 0.21 & 1315.7 & 1317.0 & 0.11 & 1164.7 & 1168.1 & 0.29 \\
\hline & 8 & 1603.8 & 1600.5 & 0.21 & 1588.2 & 1586.5 & 0.11 & 1383.3 & 1385.1 & 0.11 & 1196.1 & 1200.4 & 0.36 \\
\hline \multirow{8}{*}{ F-F } & 1 & 950.58 & 950.88 & 0.60 & 210.99 & 208.23 & 1.33 & .45 & 425.74 & 0.89 & 500.47 & 503.97 & 0.69 \\
\hline & 2 & 1227.3 & 1221.2 & 0.50 & 236.92 & 235.29 & 0.69 & 515.85 & 510.74 & 1.01 & 541.04 & 542.63 & 0.29 \\
\hline & 3 & 1271.1 & 1271.1 & 0.00 & 777.66 & 777.8 & 0.02 & 720.87 & 717.15 & 0.52 & 590.51 & 588.05 & 0.42 \\
\hline & 4 & 1355.3 & 1346.4 & 0.66 & 952.73 & 950.88 & 0.19 & 729.39 & 723.60 & 0.80 & 599.67 & 597.89 & 0.30 \\
\hline & 5 & 1454.5 & 1451.2 & 0.23 & 10 & 1036 & 0.63 & 781.02 & 779.51 & 0.20 & 875.19 & 865.99 & 1.06 \\
\hline & 6 & 1594.8 & 1591.6 & 0.20 & 1249.2 & 1247.8 & 0.11 & 956.22 & 956.7 & 0.05 & 919.06 & 912.19 & 0.75 \\
\hline & 7 & 1600.6 & 1595.5 & 0.32 & 1371.1 & 1364.8 & 0.46 & 1201.8 & 1191.3 & 0.87 & 1082.7 & 1077.2 & 0.51 \\
\hline & 8 & 1613.4 & 1606.2 & 0.45 & 1394.6 & 1383.8 & 0.78 & 1215.1 & 1206.5 & 0.76 & 1098.3 & 1090.2 & 0.74 \\
\hline
\end{tabular}




\section{Table 5}

The lowest five natural frequencies of the double-shell structure with elastic coupling boundary conditions ( $\left.L=3 \mathrm{~m}, R_{o}=0.5 \mathrm{~m}, R_{i}=0.3 \mathrm{~m}, h_{s}=h_{p}=0.005 \mathrm{~m}, x_{1}=\mathrm{L} / 2\right)$.

\begin{tabular}{|c|c|c|c|c|c|c|}
\hline \multirow{2}{*}{ E.B } & \multirow{2}{*}{\multicolumn{2}{|c|}{ Original frequency }} & \multicolumn{4}{|c|}{ Natural frequencies } \\
\hline & & & $k_{x}^{c}=10^{5}$ & $k_{\theta}^{c}=10^{5}$ & $k_{r}^{c}=10^{5}$ & $K_{r}^{c}=10^{5}$ \\
\hline \multirow{5}{*}{$\mathrm{C}-\mathrm{C}$} & 1 & 156.47 & 0.83 & 156.47 & 156.47 & 155.86 \\
\hline & 2 & 161.54 & 12.28 & 161.54 & 161.54 & 161.01 \\
\hline & 3 & 178.84 & 35.59 & 178.74 & 174.34 & 178.84 \\
\hline & 4 & 190.25 & 72.61 & 190.25 & 187.98 & 189.72 \\
\hline & 5 & 190.99 & 123.02 & 191.00 & 190.25 & 190.74 \\
\hline \multirow{5}{*}{ S-S } & 1 & 36.73 & 4.23 & 36.79 & 36.79 & 28.33 \\
\hline & 2 & 127.46 & 25.77 & 127.46 & 127.46 & 127.27 \\
\hline & 3 & 138.94 & 28.23 & 138.95 & 138.95 & 138.76 \\
\hline & 4 & 156.23 & 43.82 & 156.33 & 153.57 & 156.36 \\
\hline & 5 & 161.61 & 77.07 & 161.50 & 154.34 & 161.98 \\
\hline \multirow{5}{*}{$\mathrm{F}-\mathrm{F}$} & 1 & 12.53 & 2.23 & 4.59 & 12.53 & 9.75 \\
\hline & 2 & 15.21 & 4.23 & 12.53 & 15.21 & 14.70 \\
\hline & 3 & 36.73 & 5.13 & 13.27 & 36.73 & 23.63 \\
\hline & 4 & 38.58 & 13.99 & 15.21 & 38.58 & 28.31 \\
\hline & 5 & 41.17 & 25.77 & 36.73 & 72.30 & 38.45 \\
\hline
\end{tabular}




\section{Table 6}

The first seven natural frequencies of the double-shell structure with elastically supported end boundaries for different stiffness ( $\left.L=3 \mathrm{~m}, R_{o}=0.5 \mathrm{~m}, R_{i}=0.3 \mathrm{~m}, h_{s}=h_{p}=0.005 \mathrm{~m}, x_{1}=\mathrm{L} / 2\right)$.

\begin{tabular}{|c|c|c|c|c|c|c|c|c|}
\hline & \multirow{2}{*}{$\begin{array}{l}\text { Support } \\
\text { stiffness }\end{array}$} & \multicolumn{7}{|c|}{ Natural frequencies $(\mathrm{Hz})$} \\
\hline & & 1 & 2 & 3 & 4 & 5 & 6 & 7 \\
\hline \multirow{6}{*}{$\hat{k}_{x}$} & $10^{-6}$ & 57.96 & 66.93 & 77.40 & 81.83 & 82.27 & 108.57 & 118.47 \\
\hline & $10^{-4}$ & 57.96 & 66.93 & 77.40 & 81.83 & 82.27 & 108.57 & 118.47 \\
\hline & $10^{-1}$ & 58.91 & 68.26 & 77.66 & 84.59 & 84.61 & 108.78 & 118.54 \\
\hline & $10^{0}$ & 64.78 & 76.37 & 79.34 & 97.51 & 98.06 & 110.13 & 119.02 \\
\hline & $10^{3}$ & 79.82 & 84.52 & 96.55 & 114.49 & 114.51 & 120.75 & 126.73 \\
\hline & $10^{4}$ & 79.87 & 84.53 & 96.61 & 114.51 & 114.54 & 120.75 & 126.80 \\
\hline \multirow{6}{*}{$\hat{K}_{r}$} & $10^{-6}$ & 57.96 & 66.93 & 77.41 & 81.83 & 82.27 & 108.58 & 118.47 \\
\hline & $10^{-4}$ & 58.29 & 67.19 & 77.75 & 81.85 & 82.41 & 108.81 & 118.77 \\
\hline & $10^{-1}$ & 59.12 & 68.72 & 78.56 & 81.97 & 82.78 & 109.98 & 119.41 \\
\hline & $10^{0}$ & 59.12 & 68.73 & 78.57 & 81.97 & 82.78 & 109.99 & 119.41 \\
\hline & $10^{3}$ & 59.12 & 68.73 & 78.57 & 81.97 & 82.78 & 109.99 & 119.41 \\
\hline & $10^{4}$ & 59.12 & 68.73 & 78.57 & 81.97 & 82.78 & 109.99 & 119.41 \\
\hline \multirow{6}{*}{$\hat{k}_{r}$} & $10^{-6}$ & 57.96 & 66.93 & 77.41 & 81.83 & 82.27 & 108.58 & 118.47 \\
\hline & $10^{-4}$ & 58.53 & 67.37 & 77.87 & 81.95 & 82.60 & 108.89 & 118.78 \\
\hline & $10^{-1}$ & 131.30 & 138.90 & 142.38 & 157.78 & 158.03 & 171.42 & 182.88 \\
\hline & $10^{0}$ & 135.27 & 143.37 & 162.77 & 171.97 & 175.42 & 182.40 & 184.20 \\
\hline & $10^{3}$ & 135.72 & 143.48 & 163.30 & 172.03 & 177.52 & 184.24 & 188.03 \\
\hline & $10^{4}$ & 135.72 & 143.48 & 163.30 & 172.03 & 177.52 & 184.24 & 188.03 \\
\hline \multirow{6}{*}{$\hat{k}_{\theta}$} & $10^{-6}$ & 57.96 & 66.93 & 77.40 & 81.83 & 82.27 & 108.57 & 118.47 \\
\hline & $10^{-4}$ & 58.02 & 67.04 & 77.43 & 81.95 & 82.35 & 108.61 & 118.48 \\
\hline & $10^{-1}$ & 98.16 & 99.18 & 127.61 & 130.31 & 132.26 & 139.34 & 151.60 \\
\hline & $10^{0}$ & 130.53 & 141.05 & 159.41 & 168.89 & 170.63 & 182.86 & 185.60 \\
\hline & $10^{3}$ & 137.73 & 143.83 & 164.23 & 172.18 & 184.28 & 188.21 & 188.75 \\
\hline & $10^{4}$ & 137.73 & 143.84 & 164.23 & 172.18 & 184.28 & 188.23 & 188.76 \\
\hline
\end{tabular}

